\title{
CFD Simulation of Solid-Liquid Stirred Tanks for Low to Dense Solid Loading Systems
}

\author{
Divyamaan WADNERKAR ${ }^{a}$, Moses O. TADE ${ }^{a}$, Vishnu K. PAREEK ${ }^{a}$, Ranjeet P. UTIKAR ${ }^{a}$ ** \\ Department of Chemical Engineering, Curtin University, Perth, WA 6102 \\ *r.utikar@curtin.edu.au
}

\begin{abstract}
Numerous industrial processes require stirred tanks as mixing systems for solids suspension in liquids. Hydrodynamics of such system is critical for its design and control to improve the performance. In particular, modelling multiphase stirred tank at high solid concentration is complex due to significant particle-particle and particle-wall interactions, which are generally neglected at low concentrations in stirred tanks. Most models do not consider such interactions and deviate significantly from experimental data. Furthermore, the applicability of drag models is restricted to a limited range of conditions present in the stirred tanks. Turbulence and turbulent dispersion play a crucial role and their high accuracy is indispensable for precisely predicting the local hydrodynamics. Therefore, critical factors such as modelling approach, drag, dispersion, coefficient of restitution and turbulence are examined and discussed exhaustively in this paper. A review is presented that compares the drag models predictions and their applicability over the range of Reynolds number observed in stirred tanks. EulerEuler with kinetic theory of granular flow approach provides realistic predictions for such systems as it take the interactions of particles with particles and walls into account. Syamlal O'Brien model is found to be the most efficient drag model in the overall prediction of solid suspension. The contribution of turbulent dispersion force in improving the prediction is marginal; however, it cannot be neglected at low solid volume fractions. Reynolds stress model is shown to be a computationally viable alternative to the widely used k- $\varepsilon$ model for accurate prediction of turbulence in this turbulence-dominated system. The inferences drawn from the study and the finalised models are instrumental in accurately simulating the solid suspension in stirred tanks for a wide range of conditions. These models can be used in simulations to obtain precise results necessary for in-depth understanding of hydrodynamics in stirred tanks. Keywords: Solid-liquid Stirred Tanks, CFD, Simulation Approach, Drag model, Turbulence model
\end{abstract}




\section{Nomenclature}

$\begin{array}{ll}B_{l} & \text { blade length } \\ B_{w} & \text { blade width } \\ C & \text { impeller clearance } \\ C_{D} & \text { drag coefficient } \\ C_{D o} & \text { particle drag coefficient in still fluid } \\ D & \text { diffusivities } \\ D_{i} & \text { impeller diameter } \\ D_{s} & \text { shaft diameter } \\ d_{P} & \text { particle diameter } \\ \vec{F}_{t d} & \text { force due to turbulent dissipation } \\ \vec{F}_{q} & \text { external force } \\ \vec{F}_{l i f t} & \text { lift force } \\ \vec{F}_{v m} & \text { virtual mass force } \\ \vec{F}_{12} & \text { interphase interaction force } \\ g & \text { gravity } \\ G_{k} & \text { turbulence kinetic energy } \\ H & \text { tank height } \\ \bar{I} & \text { unit stress tensor. } \\ k & \text { turbulence kinetic energy } \\ l & \text { continuous phase } \\ m & \text { mixture properties } \\ M & \text { torque } \\ N & \text { impeller speed } \\ N_{j s} & \text { speed of just suspension } \\ N_{R e} & \text { Reynolds number }\end{array}$

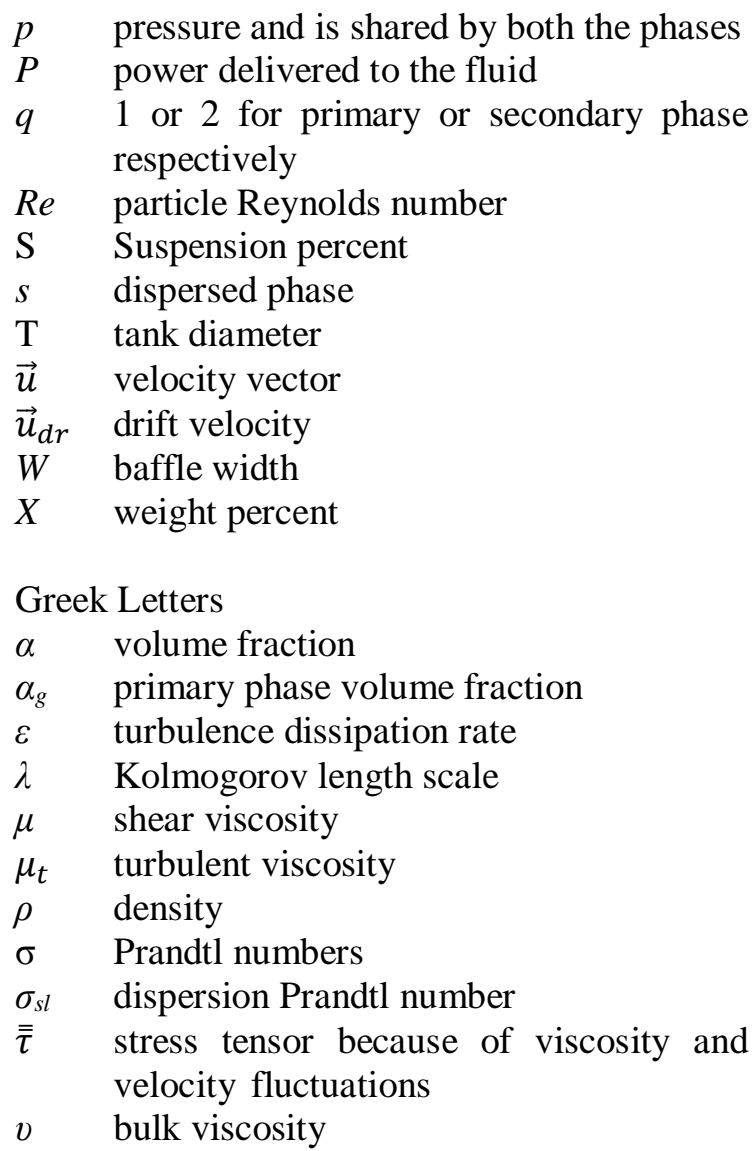

\section{Introduction}

Solid-liquid stirred tanks are widely used in mineral, chemical and pharmaceutical industries for reactions, crystallization, mass transfer operations, etc. For these processes, the efficiency of the operation depends on the contact between the constituents and suspension quality (Kasat, Khopkar, Ranade, \& Pandit, 2008). The mass transfer in a process, such as leach tanks, is facilitated by the extent of contact between the phases. However, problems such as settled solids, dead zones, accumulation of solids near the wall, dampening of the flow field, etc. cause poor interaction between the phases. Appropriate selection of design and operating parameters such as impeller clearance, tank diameter to height ratio, number of impellers, impeller speed, baffle width, etc. can minimize these problems. A detailed understanding of hydrodynamics of solid-liquid stirred tanks is indispensable for proper quantification of these parameters. Advanced multiphase flow measurement techniques such as Particle Image Velocimetry (PIV), Computer Aided Radioactive Particle Tracking (CARPT), Positron Emission Particle Tracking (PEPT), etc. have been proven to be useful in obtaining quantitative hydrodynamic data. While, these techniques work best at low solids concentration, measurements at higher solids concentrations are challenging especially with optical techniques. 
Computational Fluid Dynamics (CFD) modelling has been used successfully to understand the hydrodynamics of multiphase stirred tanks, especially at lower solids concentration (Fletcher \& Brown, 2009; Kasat et al., 2008; Tamburini, Cipollina, Micale, Brucato, \& Ciofalo, 2012, 2014). However, multiphase CFD models rely on a variety of constitutive models that needs validation, which was otherwise not possible due to unavailability of reliable data at high solid concentration. Some local data such as solid concentration and velocity can now be obtained with higher accuracy using non-intrusive techniques like CARPT, PEPT, etc. in dense multiphase stirred tanks. The data obtained can facilitate the validation of models, and these models can further be used for exhaustive investigation of hydrodynamics in stirred tanks with high solid concentrations. In this paper, a review of hydrodynamic studies of solid-liquid stirred tank systems at high solids concentration is presented. The limitations of available constitutive models in prediction of the local hydrodynamics are discussed. The two modelling approaches namely, Eulerian-Eulerian (EE) and Eulerian-Eulerian with kinetic theory of granular flow (EE-KTGF) are compared. Proper selection of interphase drag model is critical in obtaining realistic predictions. Several drag models are compared with data from direct numerical simulations. The applicability of these drag models along with turbulence and turbulent dispersion models is also examined for low to high solid loading systems.

\section{LITERATURE REVIEW}

For dense systems, opacity is a major problem due to high concentration of solids, which limits visibility. Available intrusive techniques are very sensitive with respect to the sample tube angle, sample withdrawal velocity, sample tube design, and orientation of the sample tube. In such cases, the margin of error while the collection of the samples also increases with the increase in the solid concentration (Yamazaki, Tojo, \& Miyanami, 1986). The opaqueness also renders inability to the non-intrusive optical techniques to provide accurate data for such systems. Most of the optical experimental techniques for studying the hydrodynamics such as light scattering technique (LST), laser Doppler velocimetry (LDV) and particle image velocimetry (PIV) are limited to low concentration due to their inapplicability at higher solids concentration (PiankoOprych, Nienow, \& Barigou, 2009; Unadkat, Rielly, Hargrave, \& Nagy, 2009). Therefore, examining the hydrodynamics of moderate of dense loading stirred tanks has always been a problem due to lack of tools and techniques. The consequence of this limitation is reflected in the scarce number of points for computational or experimental research for such systems as shown in Figure 1. As compared to the experimental investigations, far fewer CFD studies in high concentration are available due to the lack of experimental data for validation of models. List of computational studies for dense stirred tank systems is given in Table 1. With the emergence of experimental techniques such as CARPT and PEPT (Barigou, 2004; Guida, Nienow, \& Barigou, 2010; Stevenson et al., 2010) that are capable of resolving solid concentration and velocity data at high solid concentration, the data required for validation of CFD models of such systems has become available. The availability of data for validation, ability to resolve the local forces through CFD and inexpensive nature 
of CFD simulations will facilitate such studies that are endeavoured to gain a detailed description of multiphase flow.

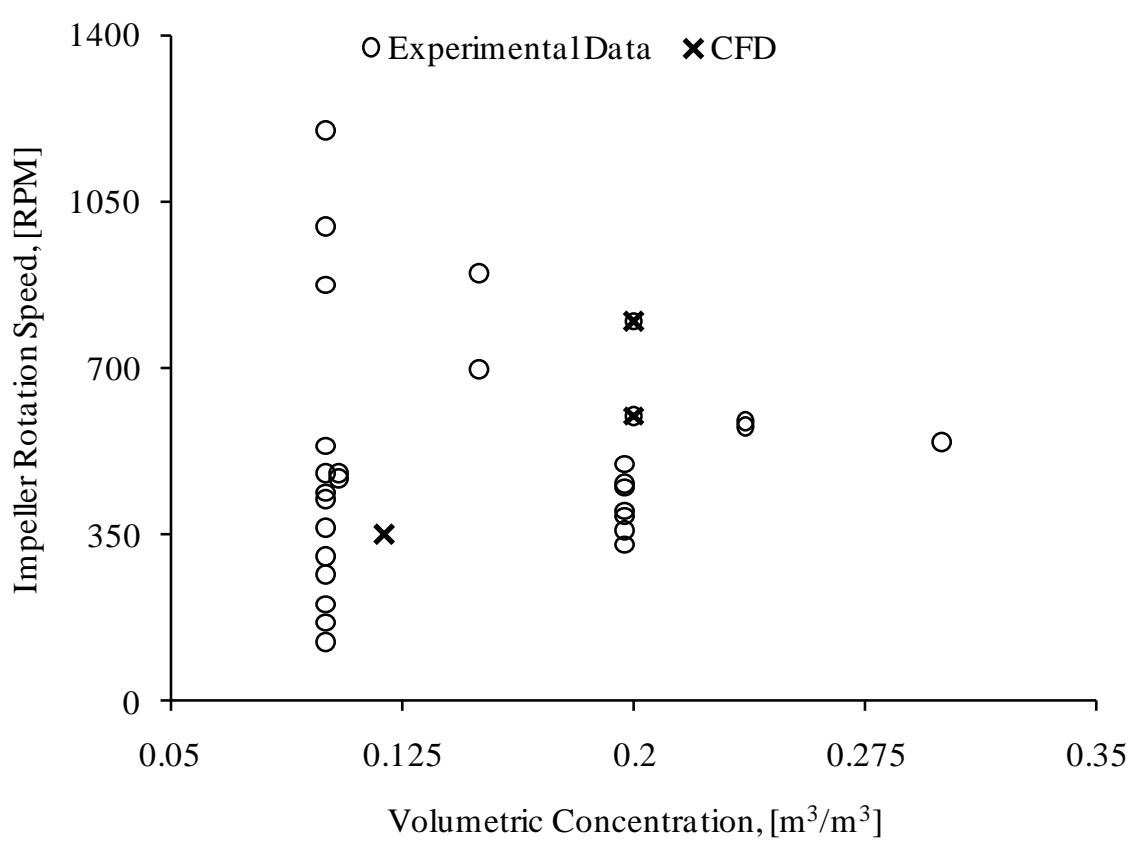

Figure 1 . The studies conducted above $10 \%$ volumetric concentration.

CFD is a powerful tool, but it requires validation of models to provide credible results. So far, the highest solid loading for which the simulation results in stirred tanks are reported is $20 \%$ (by volume) (Altway, Setyawan, Margono, \& Winardi, 2001; Micale, Grisafi, Rizzuti, \& Brucato, 2004). Altway et al. (2001) performed simulations to predict solid concentration profile in stirred tanks and validated their simulation results using data from Yamazaki et al. [2]. The models predictions matched with experimental data well for low solid concentration of $5 \%$, but major discrepancy was found in the concentration profiles of $20 \%$ solids concentration. Micale et al. (2004) used Multiple Reference Frame (MRF) and Sliding Grid (SG) approach to study the clear liquid layer and the suspension height for dense solid-liquid systems. In their simulations, the power numbers were 2.98, 2.74 and 2.68 for $\mathrm{N}=5,6.33$ and 8 RPS respectively (particle loading of $9.6 \%$ $\mathrm{v} / \mathrm{v})$, which were significantly smaller than the experimental values of $4.59,4.37$ and 4.23 . The imperfection in the solid suspension prediction was attributed to second order effects (particle drag modifications due to liquid turbulence, presence of other particles, particle-particle direct interactions, etc.) that were neglected in the study. Ochieng and Lewis (2006b), Fradette et al. (2007), Ochieng and Onyango (2008), Kasat et al. (2008), Fletcher and Brown (2009), Tamburini, Cipollina, Micale, Ciofalo, and Brucato (2009), Tamburini, Cipollina, Micale, Brucato, and Ciofalo (2011) and Tamburini et al. (2012) conducted simulations for volume fractions below $20 \%$, and validated using non-local properties like cloud height, suspension quality, etc. In another similar study, Gohel, Joshi, Azhar, Horner, and Padron (2012) used qualitative and quantitative data for cloud height to validate the simulation of high concentration solids suspension in stirred tanks. In these 
studies, while the parameters, for example cloud height, were accurately predicted, the errors in the predictions of local hydrodynamics were not verified in the absence of data. In the direction of resolving the local hydrodynamics of the stirred tanks, Liu and Barigou (2013) used local velocity field and solids concentration data for model validation and found that even though a good agreement in the axial concentration profile was observed, the local concentration predictions could still be very poor and could vary from experiments by several folds. The inaccuracy was attributed to inadequate models for particle sedimentation, lift-off and particle-particle interaction in their CFD model. They suggested incorporating particle-particle interaction in the solids pressure term given by Gidaspow (1994), but didn't use it in their models due to convergence problems.

All the available literature emphasizes on the accuracy of models for particle-particle interactions, particlefluid interactions, turbulence, turbulence dispersion, etc. for obtaining quantitative agreement on local hydrodynamics. However, there is no consensus on preferable set of these models. Ochieng and Onyango (2008) assessed different drag models for simulation of solids in stirred tanks. They found that in Stokes law region, Gidaspow model provides better results than the Brucato model, which provided best prediction in turbulent regimes. Khopkar, Kasat, Pandit, and Ranade (2006) modified the Brucato drag model by factoring the change in drag by 0.1 after determining the change of particle diameter to Kolmogorov length ratio with Reynolds number. They observed that the modified Brucato drag model performs better than Brucato drag model in predicting the solid concentration profiles. Sardeshpande, Juvekar, and Ranade (2010) also supported this finding when they used these models to compare the hysteresis in cloud heights. Similar observation is made by Wadnerkar et al. (2012) while they compared modified Brucato drag model, Brucato, Gidaspow and Wen \& Yu models for low solid holdups and particle diameter of $300 \mu \mathrm{m}$. Tamburini et al. (2014) used Brucato drag model and compared its results with the modification of Brucato drag using Wen \& Yu model and Gidaspow drag model with linear switching function, which they termed as modification by dense particle effect and piecewise correlation, respectively. Contrary to the previous findings of modified Brucato giving better results, they found that both of these modifications resulted in further deterioration in predictions and over-prediction of suspension quality. In these cases, it is worth noticing that even with the Brucato drag model, the suspension quality was over-predicted and the predictions of solid axial concentration profiles deteriorated at high stirrer speed (Reynolds number). Both modified Brucato and Brucato drag models are dependent on the ratio of particle diameter and the Kolmogorov length scale. They provide satisfactory results when the particle diameter and volume fractions are small and turbulence dominated the flow. However, the dependency of the drag model on the particle diameter makes it susceptible to over-prediction of drag force for larger particles (or high Reynolds number). In recent times, direct numerical simulations (e.g. lattice Boltzmann simulations) have provided foundation for development of drag models. Drag models derived using this method are accurate in predicting the drag force experienced by the particles (Cello, Di Renzo, \& Di Maio, 2010; Rong, Dong, \& Yu, 2013; Tenneti, Garg, \& Subramaniam, 2011). The computational 
requirement of these simulations have limited the simulations to a Reynolds number of 300 and therefore, the drag force values obtained at higher Reynolds number are extrapolated. The values from all these drag models over a range of Reynolds number need to be compared for determining the accuracy before implementation in stirred tanks system.

Another factor that influences the model predictions with increasing solids concentration is the particleparticle interactions such that their contribution cannot be neglected. Fradette et al. (2007) incorporated the particle-particle and particle fluid interactions as a function of strain tensor and volume fraction while assessing the accuracy of Shear-Induced Migration Model (SIMM) to capture the particle suspension phenomenon and particle migration in solid-liquid. They found errors in large-gap geometries because of large magnitude and opposite sign of shear rate leading to large discrepancies with respect to the experimental flow and concentration fields. J. J. Derksen (2003) emphasized on the particle-particle interaction in the hydrodynamic studies and found that by introducing particle collisions, a realistic particle distribution is obtained in the stirred tank. He used Euler-Lagrangian approach with particle collision to incorporate the effect of particle-particle interaction. This approach requires enormous computational power due to a very fine timestep of the order of $10^{-6}$ and tracking of large number of particles (ranges up to $10^{7}$ ). The associated high computational expense renders its application unfeasible using the available computational power. In this regard, Oshinowo and Bakker (2002) and Gohel et al. (2012) have used EE-KTGF approach for the modelling particle interaction in the stirred tanks, but have not discussed their specific impact on the predictions. Giuseppina Montante, Rondini, Bakker, and Magelli (2002) have presented a comparison of the predictions of using EE and EE-KTGF approach (with and without particle interaction) for the multi-impeller pitched blade turbine system, however, the analysis required further explanation and discussion on their applicability over a range of conditions in stirred tanks (especially at high solids concentration) on the involved particleparticle interaction parameters, for example restitution coefficient.

The choice of model for turbulent dispersion force and turbulence strongly affect prediction of solid suspension (Gohel et al., 2012; Khopkar et al., 2006; Ljungqvist \& Rasmuson, 2001; Tamburini et al., 2014). Use of turbulent dispersion force was shown to have significantly better predictions of cloud height and solids concentration profiles (Gohel et al., 2012). The turbulent dispersion was studied at low solids concentration by Ochieng and Lewis (2006b) who incorporated the models of de Bertodano, Lahey Jr, and Jones (1994) (DBL) and Burns, Frank, Hamill, and Shi (2004). They found little influence of dispersion force calculated using DBL model on the predictions, and pointed that using the force reduced the computation time by $20 \%$. However, they did not report the findings using Burns model. Proper assessment of the influence of turbulent dispersion on solids suspension is not available. Turbulence models, on the other hand, are important and are extensively studied in case of single phase flows (Aubin, Fletcher, \& Xuereb, 2004), and multiphase flows (G. Montante \& Magelli, 2005). Aubin et al. (2004) compared the predictions of standard k- $\varepsilon$ and Renormalised Group (RNG) k- $\varepsilon$. They found that the turbulent kinetic energy is grossly under-predicted in the 
impeller discharge region due to anisotropic nature of flow that cannot be resolved with the isotropic assumption in k- $\varepsilon$ standard and RNG models. However, they were not able to quantify it using the Reynolds Stress Model (RSM), which is not based on isotropic assumption, due to convergence problems in their solution. G. Montante and Magelli (2005) investigated different variations of k- $\varepsilon$ turbulence models, namely, mixture, per phase and dispersed models for simulating stirred tanks with upto $6 \%$ solids by volume. Although the mixture and per phase k- $\varepsilon$ model provided better predictions than the dispersed model, but the deviation from the experimental data was not eliminated by using either of these models. Feng, Li, Cheng, Yang, and Mao (2012) emphasized on resolving the anisotropic nature of turbulence for improving the predictions. They extended the single phase explicit algebraic slip model (EASM) to the multiphase stirred tank system and found that this model is superior to the standard k- $\varepsilon$ turbulence model. However, the predictability of solid concentration using EASM deteriorated for high solid concentration stirred tank systems. J. Derksen, Akker, and Harry (1999) evaluated Large Eddy Simulations (LES) and successfully resolved the anisotropic turbulence, trailing vortex and phase resolved velocity fields in the stirred tanks. However, such simulations require immense computational power that can be 100 times higher than that used to perform EE simulations with seven equations RSM model.

Table 1. Details of computational studies conducted for moderate to dense solid loading systems.

\begin{tabular}{|c|c|c|c|c|c|}
\hline Author and year & Solid Loading & $\begin{array}{l}\text { Impeller } \\
\text { Speed }\end{array}$ & Techniques & Geometry & Impeller \\
\hline & $\begin{array}{l}\% \mathrm{v} / \mathrm{v} \text { or } \% \mathrm{w} / \mathrm{w} \\
\text { or } \mathrm{g} / \mathrm{l}\end{array}$ & (in RPM) & & Tank Type & Impeller Type \\
\hline $\begin{array}{l}\text { Altway et al. } \\
(2001)\end{array}$ & 5 and $20 \% \mathrm{v} / \mathrm{v}$ & 800 & Fluent; Algebraic Slip Model; & $\begin{array}{l}\text { Flat-bottom } \\
\text { cylindrical tank }\end{array}$ & Rushton Turbine \\
\hline $\begin{array}{l}\text { Micale et al. } \\
(2004)\end{array}$ & $\begin{array}{l}0.48,2.4,4.8,9.6 \\
\text { and } 20 \% \mathrm{v} / \mathrm{v}\end{array}$ & $\begin{array}{l}300, \quad 380 \\
\text { and } 480\end{array}$ & $\begin{array}{l}\text { Ansys CFX 4.4: } \\
\text { Eulerian-eulerian approach }\end{array}$ & $\begin{array}{ll}\text { Flat } & \text { bottom } \\
\text { Cylindrical Vessel }\end{array}$ & Rushton Turbine \\
\hline $\begin{array}{l}\text { Ochieng and } \\
\text { Lewis (2006b) }\end{array}$ & $1-20 \% \mathrm{w} / \mathrm{w}$ & $\begin{array}{l}200-700 \\
\text { RPM }\end{array}$ & $\begin{array}{l}\text { CFX: } \\
\text { Sliding Grid approach; }\end{array}$ & $\begin{array}{l}\text { Elliptical bottom } \\
\text { Cylindrical Vessel }\end{array}$ & $\begin{array}{l}\text { Four blade Mixtec } \\
\text { HA735 propeller }\end{array}$ \\
\hline $\begin{array}{l}\text { Ochieng and } \\
\text { Lewis (2006a) }\end{array}$ & $1-20 \% \mathrm{w} / \mathrm{w}$ & $200-700$ & $\begin{array}{l}\text { CFX 5.6/5.7: } \\
\text { Eulerian-Eulerian approach }\end{array}$ & $\begin{array}{l}\text { Elliptical bottom } \\
\text { Cylindrical Vessel }\end{array}$ & $\begin{array}{l}\text { Four } \quad r \text { Blade } \\
\text { Hydrofoil Propeller } \\
\text { (MIXTEC HA735) } \\
\end{array}$ \\
\hline \multirow{2}{*}{$\begin{array}{l}\text { Fradette et al. } \\
(2007)\end{array}$} & $2.8 \% \mathrm{v} / \mathrm{v}$ & 173 & \multirow[t]{2}{*}{ Analytical: SIMM model } & \multirow{2}{*}{$\begin{array}{ll}\text { Flat } & \text { bottom } \\
\text { Cylindrical Vessel }\end{array}$} & \multirow{2}{*}{$\begin{array}{l}\text { Down-pumping } \\
\text { Marine Propeller }\end{array}$} \\
\hline & $\begin{array}{l}7.1 \% \mathrm{v} / \mathrm{v} \\
11.9 \% \mathrm{v} / \mathrm{v}\end{array}$ & $\begin{array}{l}230 \\
350\end{array}$ & & & \\
\hline $\begin{array}{l}\text { Ochieng and } \\
\text { Onyango (2008) }\end{array}$ & $1-20 \% \mathrm{w} / \mathrm{w}$ & $200-700$ & $\begin{array}{l}\text { CFX; Multiple frames of } \\
\text { Reference and Sliding Grid } \\
\text { approach }\end{array}$ & $\begin{array}{ll}\text { Flat } & \text { bottom } \\
\text { Cylindrical } & \text { Vessel }\end{array}$ & $\begin{array}{l}\text { Four blade Mixtec } \\
\text { HA735 propeller }\end{array}$ \\
\hline $\begin{array}{l}\text { Kasat et al. } \\
(2008)\end{array}$ & $10 \% \mathrm{v} / \mathrm{v}$ & $120-2400$ & $\begin{array}{l}\text { CFD Simulation: } \\
\text { Fluent } \\
\text { Eulerian-eulerian approach. }\end{array}$ & $\begin{array}{ll}\text { Flat } & \text { bottom } \\
\text { Cylindrical } & \text { Vessel }\end{array}$ & Rushton Turbine \\
\hline $\begin{array}{l}\text { Fletcher and } \\
\text { Brown (2009) }\end{array}$ & $9.6 \% \mathrm{v} / \mathrm{v}$ & $\begin{array}{l}240, \quad 420 \\
\text { and } 600\end{array}$ & $\begin{array}{l}\text { ANSYS CFX } 11 \\
\text { Eulerian-Euleriam Model } \\
\text { Algebraic Slip Modelling } \\
\text { (ASM) }\end{array}$ & $\begin{array}{ll}\text { Flat } & \text { bottom } \\
\text { Cylindrical } & \text { Vessel }\end{array}$ & Rushton Turbine \\
\hline $\begin{array}{l}\text { Tamburini et al. } \\
(2011, \quad 2012 ; \\
2009)\end{array}$ & $\begin{array}{l}5.95 \%, \quad 9.2 \%, \\
9.6 \%, 11.9 \% \mathrm{v} / \mathrm{v}\end{array}$ & $\begin{array}{ll}300 & \text { to } \\
1200 & \end{array}$ & $\begin{array}{l}\text { Ansys-CFX4.4; } \\
\text { Eulerian MFM; }\end{array}$ & $\begin{array}{ll}\text { Flat } & \text { bottom } \\
\text { Cylindrical } & \text { Vessel }\end{array}$ & Rushton Turbine \\
\hline $\begin{array}{l}\text { Liu and Barigou } \\
(2013)\end{array}$ & $\begin{array}{l}2.5 \%, 5.2 \% \text { and } \\
10.4 \% \mathrm{v} / \mathrm{v}\end{array}$ & $360-735$ & ANSYS CFX 12.0 & $\begin{array}{ll}\text { Flat } & \text { bottom } \\
\text { Cylindrical } & \text { Vessel } \\
\end{array}$ & $\begin{array}{l}\text { Pitched } \\
\text { Turbine }\end{array}$ \\
\hline
\end{tabular}


From the review presented so far, following conclusions can be deduced:

1) Most of the simulations are conducted at low solids concentration due to unavailability of experimental data at higher solids concentration.

2) The validation of simulation using global parameters such as cloud height or per cent suspension provides little or no information on the accuracy of local hydrodynamics.

3) Deviation of the predictions from experimental observations is attributed to either lack or accuracy of models like particle-particle interaction, fluid-particle interaction, turbulence, etc.

4) Models for the particle-fluid interaction, particle-particle interaction and turbulence are applicable for a limited range of conditions. In a stirred tank multiphase system with complex flow field involving various flow regimes, the applicability of the models heavily depend on the conditions prevailing in the tanks, and may not be applicable for a different condition such as higher solids concentration, larger particle diameter, etc.

In order to resolve these issues, it is necessary to quantify the influence of various models on the accuracy of the prediction of local hydrodynamics of the system for a range of conditions. In this paper, Eulerian multiphase simulations are used to investigate the hydrodynamics in a stirred tank for low to dense solids concentration. The CFD simulation results are compared with the experimental solids concentration data obtained from PEPT measurement of the stirred tanks (Guida et al., 2010). This experimental data set is used as it presents both axial and radial concentration profiles of solids. The simulation results are presented and analysed for a range of solid concentrations representing low, medium and high solids concentration. For characterizing the dynamics of stirred tanks at high solids concentration, key information such as effect of modelling approach, constitutive parameters such as coefficient of restitution (for particle-particle interaction), drag force (for particle-fluid interaction), dispersion force, and turbulence are presented and examined extensively. Applicability of the models for different flow regimes and solids concentrations is discussed. This information is useful for accurately modelling of solid-loading suspensions and evaluation of mixing efficiency of an impeller.

\section{Model Description}

\section{Governing Equations}

The hydrodynamic simulations are conducted using Eulerian-Eulerian multiphase model. In this model, each phase is treated as an interpenetrating continuum represented by a volume fraction at each point of the system. Reynolds averaged mass and momentum balance equations are solved for each phase. The governing equations are:

Continuity equation: 


$$
\frac{\partial}{\partial t}\left(\alpha_{q} \rho_{q}\right)+\nabla \cdot\left(\alpha_{q} \rho_{q} \bar{u}_{q}\right)=0
$$

Momentum equation:

$$
\frac{\partial}{\partial t}\left(\alpha_{q} \rho_{q} \vec{u}_{q}\right)+\nabla \cdot\left(\alpha_{q} \rho_{q} \vec{u}_{q} \vec{u}_{q}\right)=-\alpha_{q} \nabla p+\nabla \cdot \bar{\tau}_{q}+\alpha_{q} \rho_{q} \vec{g}+\vec{F}_{t d, q, v m, l i f t}+\vec{F}_{12}
$$

For the sake of brevity, only the equations of standard k- $\varepsilon$ model for turbulence are given below.

$$
\begin{aligned}
& \frac{\partial}{\partial t}\left(\rho_{m} k\right)+\nabla \cdot\left(\rho_{m} \vec{u}_{m} k\right)=\nabla \cdot\left(\mu_{m}+\frac{\mu_{t, m}}{\sigma_{k}} \nabla k\right)+G_{k, m}+G_{b, m}-\rho_{m} \varepsilon \\
& \frac{\partial}{\partial t}\left(\rho_{m} \varepsilon\right)+\nabla \cdot\left(\rho_{m} \vec{u}_{m} \varepsilon\right)=\nabla \cdot\left(\frac{\mu_{t, m}}{\sigma_{\varepsilon}} \nabla \varepsilon\right)+\frac{\varepsilon}{k}\left(C_{1 \varepsilon} G_{k, m}-C_{2 \varepsilon} \rho_{m} \varepsilon\right)
\end{aligned}
$$

where $\mathrm{C}_{1 \varepsilon}$ and $\mathrm{C}_{2 \varepsilon}$ are constants.

And, $\quad \rho_{m}=\sum_{i=1}^{N} \alpha_{i} \rho_{i}$

$$
\vec{u}_{m}=\frac{\sum_{i=1}^{N} \alpha_{i} \rho_{i} \vec{u}_{i}}{\sum_{i=1}^{N} \alpha_{i} \rho_{i}}
$$

Eddy viscosity is computed from

$$
\mu_{t, m}=\rho_{m} C_{\mu} \frac{k^{2}}{\varepsilon}
$$

Evaluation of generation of turbulent kinetic energy is consistent with Boussinesq hypothesis and is computed as

$$
G_{k, m}=\mu_{t, m}\left(\nabla \vec{u}_{m}+\nabla \vec{u}_{m}^{T}\right): \nabla \vec{u}_{m}
$$

The equation of conservation of granular temperature is given as:

$$
\frac{3}{2}\left[\frac{\partial}{\partial \mathrm{t}}\left(\alpha_{\mathrm{s}} \rho_{\mathrm{s}} \Theta_{\mathrm{s}}\right)+\nabla \cdot\left(\alpha_{\mathrm{s}} \rho_{\mathrm{s}} \overrightarrow{\mathrm{u}}_{\mathrm{s}} \Theta_{\mathrm{s}}\right)\right]=-\left(\mathrm{p}_{\mathrm{s}} \overline{\overline{\mathrm{I}}}+\nabla \cdot \overrightarrow{\bar{\tau}} \overline{\mathrm{s}}_{\mathrm{s}}\right): \nabla \overrightarrow{\mathrm{u}}_{\mathrm{s}}+\nabla \cdot\left(\mathrm{k}_{\Theta_{\mathrm{s}}} \nabla \Theta_{\mathrm{s}}\right)-\gamma_{\Theta_{\mathrm{s}}}+\phi_{\mathrm{ls}}
$$

where $-\left(\mathrm{p}_{\mathrm{s}}^{\overline{\mathrm{I}}}+\nabla \cdot \bar{\tau}_{\mathrm{s}}\right): \nabla \overrightarrow{\mathrm{u}}_{\mathrm{q}}$ is the generation of energy by the solid stress tensor, $\nabla \cdot\left(\mathrm{k}_{\Theta_{\mathrm{s}}} \nabla \Theta_{\mathrm{s}}\right)$ is the diffusion of energy, $\gamma_{\Theta_{\mathrm{s}}}$ is the collisional dissipation of energy and $\phi_{\mathrm{ls}}$ is the energy exchange between fluid and solid phase.

Table 2. Constitutive Equations. 


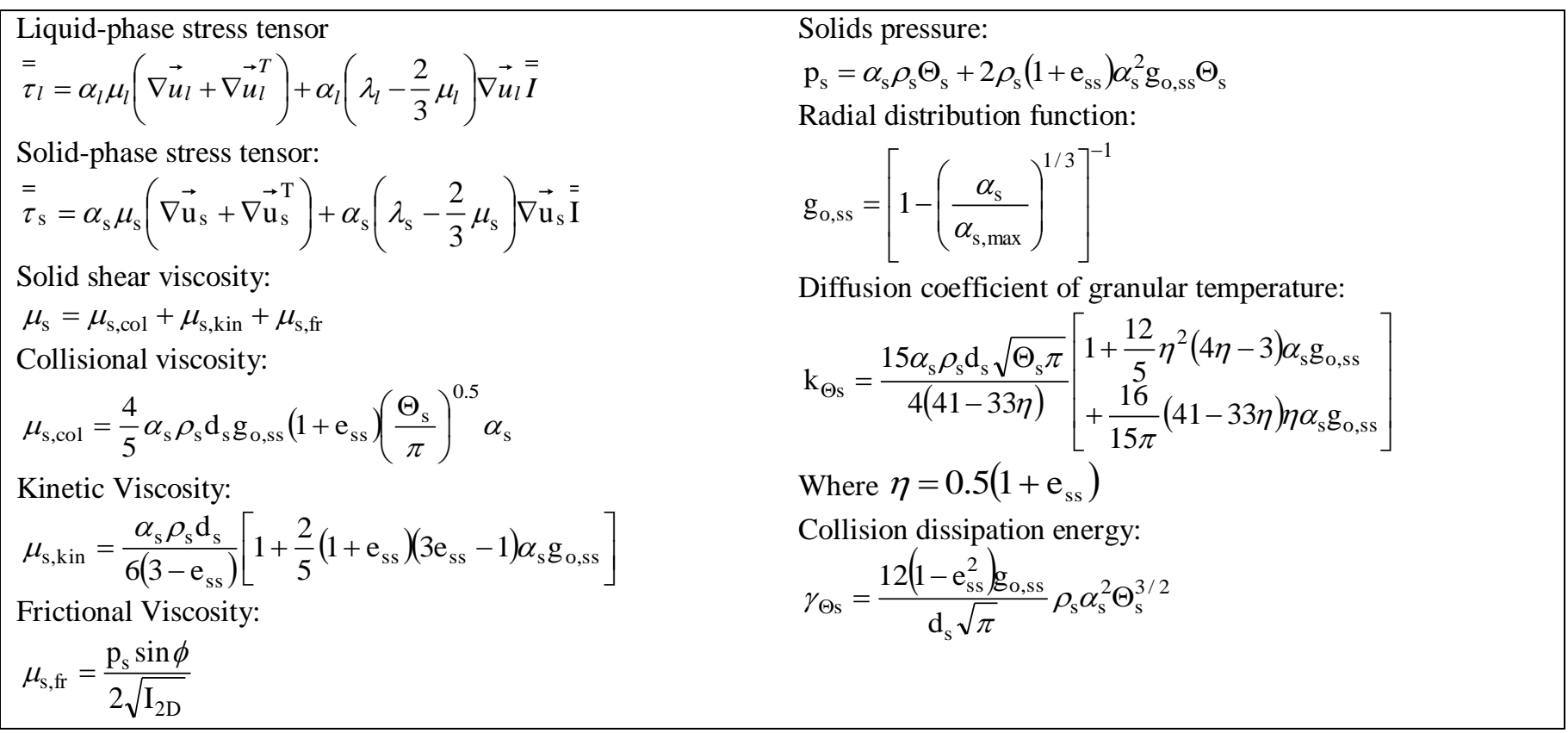

The stress-strain tensor in the momentum transfer equation is due to viscosity and Reynolds stresses that include the effect of turbulent fluctuations. Boussinesq's eddy viscosity hypothesis is used for the closure of momentum transfer equation. The particle-particle interaction is modelled using kinetic theory of granular flow by assuming that its behaviour similar to dense gas. Similar to the thermodynamic temperature of gases, the granular temperature is used to model the fluctuating velocity of particles (Gidaspow, 1994). The inclusion of lift force and virtual mass force is found to have negligible effect on the predictions in solid-liquid stirred tank simulations (Ljungqvist \& Rasmuson, 2001). Ljungqvist and Rasmuson (2001) observed it while investigating the influence of these forces on the prediction of slip velocities. Therefore, in the simulations presented in the paper, both of these forces are neglected.

\section{Turbulence}

Turbulence is not resolved in the RANS simulations and therefore, it needs to be modelled. For modelling turbulence, zero equation turbulence model (e.g. mixing length), one equation models (e.g. Spalart-Allmaras model), two equation models (e.g. k- $\varepsilon, \mathrm{k}-\omega$ and their variants, etc.), Reynolds Stress models (RSM), Large Eddy Simulations (LES), etc. are used. Although LES resolves the larger anisotropic turbulence scales, it requires simulation of complete domain with fine mesh, which is very computationally expensive (J. Derksen et al., 1999). G. Montante and Magelli (2005) compared the extensions of k- $\varepsilon$ model and found that 'mixture' and 'each phase' models provided similar results, but results of 'dispersed' model were rather unrealistic. Standard k- $\varepsilon$ is the most commonly used model in the RANS simulations of stirred tanks (Altway et al., 2001; Fan, Mao, \& Wang, 2005; Kasat et al., 2008; Khopkar et al., 2006; Ljungqvist \& Rasmuson, 2001; Micale, Montante, Grisafi, Brucato, \& Godfrey, 2000; G. Montante \& Magelli, 2005). However, it finds limitation in modelling anisotropic turbulence in the impeller discharge region and under-predicts turbulent kinetic energy in flow impingement region. RSM model predicts the Reynolds stresses by explicitly solving their governing equations. Hence, it resolves the anisotropic turbulence and may result in improved predictions of turbulence 
in such regions. Therefore, in this paper, the results of solids suspension in stirred tanks using variants of k- $\varepsilon$ and k- $\omega$ models are compared with that of RSM model.

\section{Turbulent Dispersion Force}

Turbulent fluctuations result in dispersion of phases from high volume fraction regions to low volume fraction regions. The turbulent dispersion force is significant when the size of turbulent eddies is larger than the particle size (Kasat et al., 2008). The contribution of turbulence dispersion in the accurate prediction of hydrodynamics in stirred tanks is assessed and the cases are discussed in the paper. Three turbulent dispersion force model are evaluated in the study viz. de Bertodano et al. (1994), Simonin and Viollet (1990), and Burns et al. (2004) models. The model equations for these three models are given below:

$$
\begin{array}{ll}
\text { de Bertodano et al. (1994) } & \overrightarrow{\mathrm{F}}_{\mathrm{td}, \mathrm{q}}=\mathrm{C}_{\mathrm{TD}} \rho_{\mathrm{q}} \mathrm{k}_{\mathrm{q}} \nabla \alpha_{\mathrm{p}} \\
\text { Simonin and Viollet (1990) } & \overrightarrow{\mathrm{F}}_{\mathrm{td}, \mathrm{q}}=\mathrm{C}_{\mathrm{TD}} \mathrm{k}_{\mathrm{pq}} \frac{\mathrm{D}_{\mathrm{t}, \mathrm{pq}}}{\sigma_{\mathrm{pq}}}\left(\frac{\nabla \alpha_{\mathrm{p}}}{\alpha_{\mathrm{p}}}-\frac{\nabla \alpha_{\mathrm{q}}}{\alpha_{\mathrm{q}}}\right) \\
\text { Burns et al. (2004) } & \overrightarrow{\mathrm{F}}_{\mathrm{td}, \mathrm{q}}=\mathrm{C}_{\mathrm{TD}} \mathrm{k}_{\mathrm{pq}} \frac{\mathrm{D}_{\mathrm{t}, \mathrm{q}}}{\sigma_{\mathrm{pq}}}\left(\frac{\nabla \alpha_{\mathrm{p}}}{\alpha_{\mathrm{p}}}-\frac{\nabla \alpha_{\mathrm{q}}}{\alpha_{\mathrm{q}}}\right)
\end{array}
$$

\section{Interphase drag force}

Interphase drag is the resultant force experienced by the particle in the direction of relative motion due to a moving fluid. Since, the solids and liquid phases are treated as interpenetrating, an inter-phase momentum exchange term is required. The accurate value of drag force experienced by an array of spheres was evaluated by Lattice Boltzmann Method (LBM) simulations for the particle Reynolds number upto 120 (Beetstra, van der Hoef, \& Kuipers, 2007). Tenneti et al. (2011) used Direct Numerical Simulations (DNS) simulations to evaluate drag for Reynolds number upto 300 for random arrangement of particles. Rong has given a new expression of $\eta$ in the expression for drag model, which is a modification of Di Felice Drag. The results of Beetstra et al. (2007), Tenneti et al. (2011) and Rong et al. (2013) are the same for Reynolds number up to 120, after which the drag calculated by expression given by Beetstra et al. (2007) and Rong et al. (2013) deviated from the DNS simulation values of drag obtained by Tenneti et al. (2011), particularly for high solid fractions. The drag correlations viz. Cello, Rong, Huilin-Gidaspow (H-G), Gibilaro, Di-Felice, Syamlal \& Brian (S\&B) and modified Brucato (M. B.)(Khopkar et al., 2006) are compared by taking data of Tenneti et al. (2011) as reference over the range of Reynolds numbers experienced in stirred tanks (see Figure 2).

For comparing the drag force, its values is normalised by using the expression:

$$
\mathrm{F}_{\mathrm{d}, \text { Normalised }}=\frac{\mathrm{F}_{\mathrm{d}}}{3 \pi \mu \mathrm{d}_{\mathrm{p}} \mathrm{U}_{\mathrm{s}}}
$$

The expressions for the normalised drag force obtained are tabulated in Table 3.

Table 3. Expressions of normalised drag. 


\begin{tabular}{|c|c|}
\hline $\begin{array}{l}\text { Huilin-Gidaspow (Huilin, } \\
\text { Gidaspow, Bouillard, \& Wentie, } \\
\text { 2003) }\end{array}$ & $\begin{array}{ll}\frac{150}{18} \frac{\left(1-\alpha_{\mathrm{g}}\right)}{\alpha_{\mathrm{g}}^{2}}+\frac{1.75}{18} \frac{\mathrm{Re}}{\alpha_{\mathrm{g}}^{2}} & \text { for } \alpha_{\mathrm{g}}>0.2 \\
\left(1+0.15 \mathrm{Re}^{0.687}\right) \alpha_{\mathrm{g}}^{-3.65} & \text { for } \alpha_{\mathrm{g}}<0.2\end{array}$ \\
\hline $\begin{array}{l}\text { Gibilaro (Gibilaro, Di Felice, } \\
\text { Waldram, \& Foscolo, 1985) }\end{array}$ & $(0.961+0.0186 \mathrm{Re}) \alpha_{\mathrm{g}}^{-3.8}$ \\
\hline Di-Felice (Felice, 1996) & $\frac{\operatorname{Re}}{24}\left(0.63+\frac{4.8}{\sqrt{\operatorname{Re}}}\right) \alpha_{\mathrm{g}}^{-\eta} \quad$ where, $\eta=3.7-0.65 \exp \left(-\frac{(1.5-\log \operatorname{Re})^{2}}{2}\right)$ \\
\hline Rong (Rong et al., 2013) & 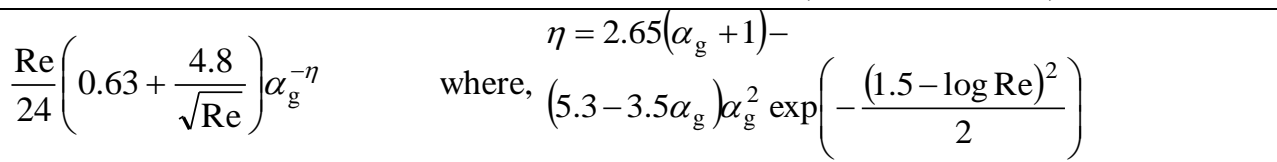 \\
\hline $\begin{array}{l}\text { Syamlal and Brian (Syamlal, } \\
\text { Rogers, \& O'Brien, 1993) }\end{array}$ & 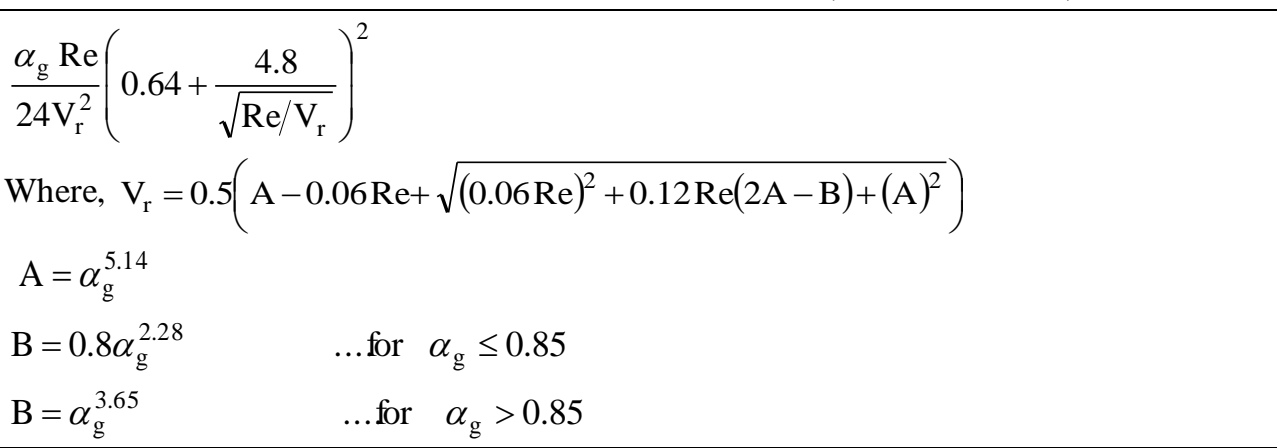 \\
\hline $\begin{array}{l}\text { Modified Brucato (Khopkar et } \\
\text { al., 2006) }\end{array}$ & $8.76 \times 10^{-5}\left(\frac{\mathrm{d}_{\mathrm{p}}}{\lambda}\right)^{3}\left(1+0.15 \mathrm{Re}^{0.687}\right) \alpha_{\mathrm{g}}^{-3.65}$ \\
\hline Cello (Cello et al., 2010) & $\begin{array}{l}\mathrm{K}_{1}+\mathrm{K}_{2} \alpha_{\mathrm{g}}^{4}+\mathrm{K}_{3}\left(1-\alpha_{\mathrm{g}}^{4}\right) \text { where, } \\
\mathrm{K}_{0}=\frac{1-\alpha_{\mathrm{g}}}{1+3 \alpha_{\mathrm{g}}} \\
\mathrm{K}_{1}=\frac{1+128 \mathrm{~K}_{0}+715 \mathrm{~K}_{0}^{2}}{\alpha_{\mathrm{g}}^{2}\left(1+49.5 \mathrm{~K}_{0}\right)} \\
\mathrm{K}_{2}=\frac{1+0.130 \mathrm{Re}+6.66 \times 10^{-4} \mathrm{Re}^{2}}{1+0.0342 \mathrm{Re}+6.92 \times 10^{-6} \mathrm{Re}^{2}}-1 \\
\mathrm{~K}_{3}=\left(\frac{2 \operatorname{Re}^{2}}{1+\operatorname{Re}}\right)\left(\frac{-410 \alpha_{\mathrm{g}}+9.20 \times 10^{7} \operatorname{Re~K}_{0}^{20}+1900 \alpha_{\mathrm{g}}^{2}-0.066 \mathrm{Re}}{6600 \alpha_{\mathrm{g}}+4.92 \times 10^{-4} \operatorname{Re}-4.30 \times 10^{4} \alpha_{\mathrm{g}}^{2}-1.31 \times 10^{-4} \operatorname{Re}^{2}+7.38 \times 10^{4} \alpha_{\mathrm{g}}^{3}}\right.\end{array}$ \\
\hline Tenneti (Tenneti et al., 2011) & $\frac{1+0.15 \operatorname{Re}^{0.687}}{\alpha_{\mathrm{g}}^{2}}+\frac{5.81\left(1-\alpha_{\mathrm{g}}\right)}{\alpha_{\mathrm{g}}^{2}}+\frac{0.48\left(1-\alpha_{\mathrm{g}}\right)^{1 / 3}}{\alpha_{\mathrm{g}}^{3}}+\left(1-\alpha_{\mathrm{g}}\right)^{\beta} \alpha_{\mathrm{g}} \operatorname{Re}\left(0.95+0.61 \frac{\left(1-\alpha_{\mathrm{g}}\right)^{3}}{\alpha_{\mathrm{g}}^{2}}\right)$ \\
\hline
\end{tabular}

The drag force predicted by different models differs particularly at high Reynolds numbers and is a major cause of discrepancy in the predictions in stirred tanks. Significant increase in drag force with increasing turbulence was reported by Brucato et al. (1998) and Khopkar et al. (2006) and a correlation was proposed that depends on the ratio of particle diameter and Kolmogorov length scale. They validated the drag models for particle diameters upto $500 \mu \mathrm{m}$. In the cases presented in the study, the coarse particles of $3000 \mu \mathrm{m}$ diameter are used that render a substantial increase in the drag force compared to that calculated at $500 \mu \mathrm{m}$. The discrepancy in the predicted drag and their currently known applicability range suggest the requirement 
of evaluation of drag models in stirred tanks. In the current study, the simulations using modified Brucato (M. B.), Huilin-Gidapow, Syamlal and Tenneti drag model are presented and compared.
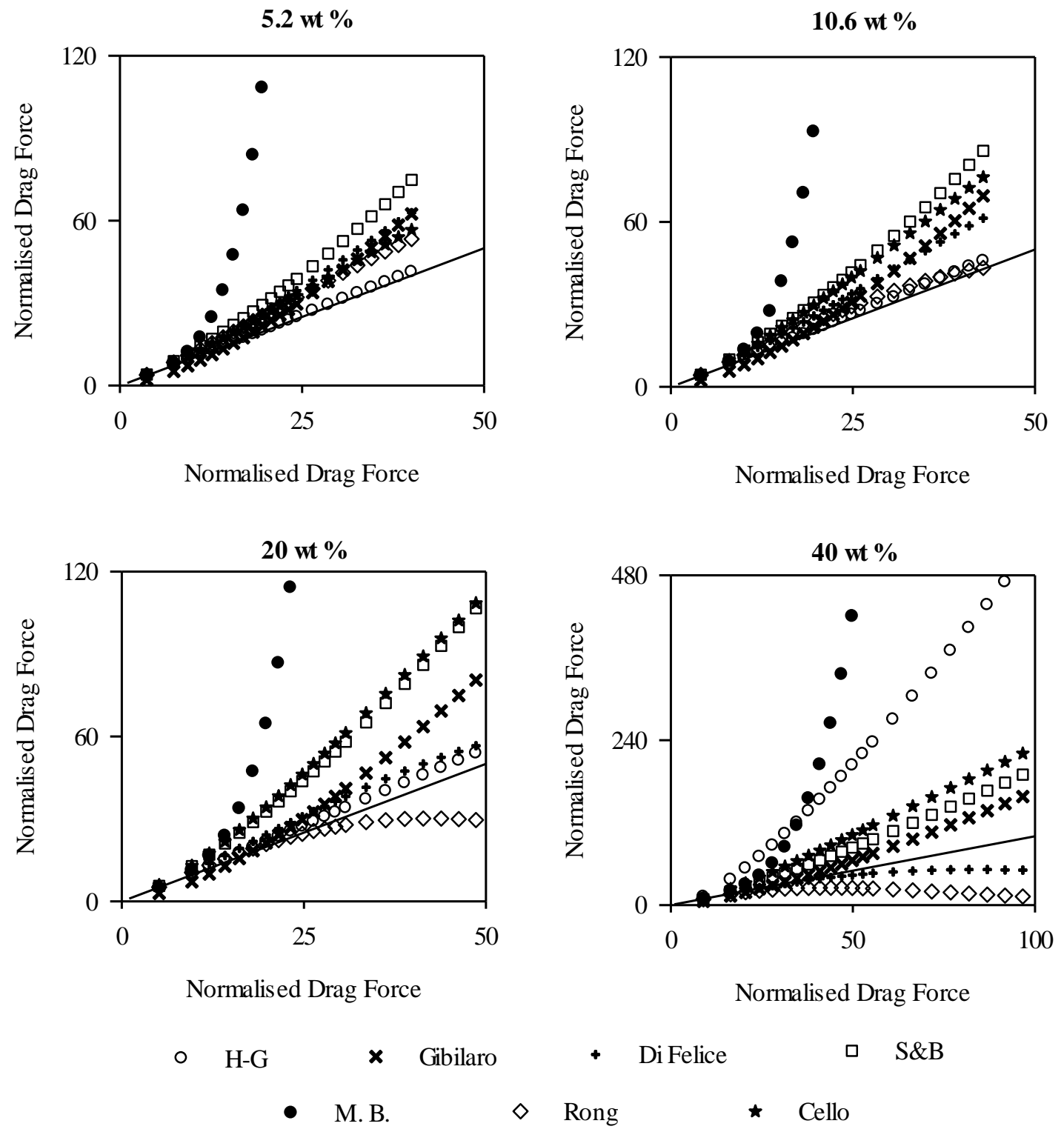

Figure 2. Parity plot for the comparison of drag forces with Tenneti drag force for Reynolds numbers upto 3000 at 5.2 wt $\%, 10.6$ wt $\%, 20 \mathrm{wt} \%$ and $40 \mathrm{wt} \%$ solid loading using different drag models.

\section{METHODOLOGY AND BOUNDARY CONDITIONS}

\section{Vessel geometry}

In this paper, a flat bottomed cylindrical tank was simulated (see Figure 3). The shaft of the impeller was concentric with the axis of the tank. A $45^{\circ}$ six-bladed Pitched Blade Turbine pumping downwards (PBTD) was used as an impeller. Particle properties reported in the experimental data are used in the simulations. The dimensions of tank and impeller are given in Table 4. The fluid and particle properties used in the simulation 
are also tabulated in the same table. Conditions such as solid concentrations, impeller speed and the Reynolds number in the tank are tabulated in Table 5. Simulations were carried out at just suspension speed for each case, which was determined by Guida et al. (2010) following Zwietering (1958) criterion. According to this criterion, no particle should remain stationary on the base of the vessel for longer than 1-2 s.

Table 4. Dimensions of domain and properties of materials used in this study.

\begin{tabular}{|ll|ll|ll|}
\hline Tank (in $\mathrm{m}$ ) & & PBTD (in $\mathrm{m})$ & & Material \\
\hline $\mathrm{T}$ & 0.288 & D & 0.144 & $\rho_{1}$ & $1150 \mathrm{~kg} / \mathrm{m}^{3}$ \\
\hline $\mathrm{H}$ & 0.288 & $\mathrm{~B}_{\mathrm{l}}$ & 0.055 & $\mu_{1}$ & $0.001 \mathrm{~Pa} . \mathrm{s}$ \\
\hline $\mathrm{W}$ & 0.0288 & $\mathrm{~B}_{\mathrm{w}}$ & 0.041 & $\rho_{\mathrm{p}}$ & $2585 \mathrm{~kg} / \mathrm{m}^{3}$ \\
\hline $\mathrm{C}$ & 0.072 & $\mathrm{D}_{\text {shaft }}$ & 0.01 & $\mathrm{~d}_{\mathrm{p}}$ & $0.003 \mathrm{~m}$ \\
\hline & & Dhub & 0.034 & $\mathrm{X}$ & $5.2,10.6,20$ and $40 \mathrm{wt} \%$ \\
& & & & & \\
\hline
\end{tabular}

Table 5. Conditions in stirred tanks used for simulations.

\begin{tabular}{|l|l|l|l|l|l|}
\hline $\mathrm{X}(\mathrm{wt} \%)$ & $\mathrm{N}=\mathrm{N}_{\mathrm{js}}(\mathrm{RPM})$ & $\mathrm{N}_{\mathrm{Re}}$ & $\mathrm{X}(\mathrm{wt} \%)$ & $\mathrm{N}=\mathrm{N}_{\mathrm{js}}(\mathrm{RPM})$ & $\mathrm{N}_{\mathrm{Re}}$ \\
\hline 5.2 & 360 & 143078.4 & 20 & 480 & 190771.2 \\
\hline 10.6 & 405 & 160963.2 & 40 & 589.8 & 234410.1 \\
\hline
\end{tabular}

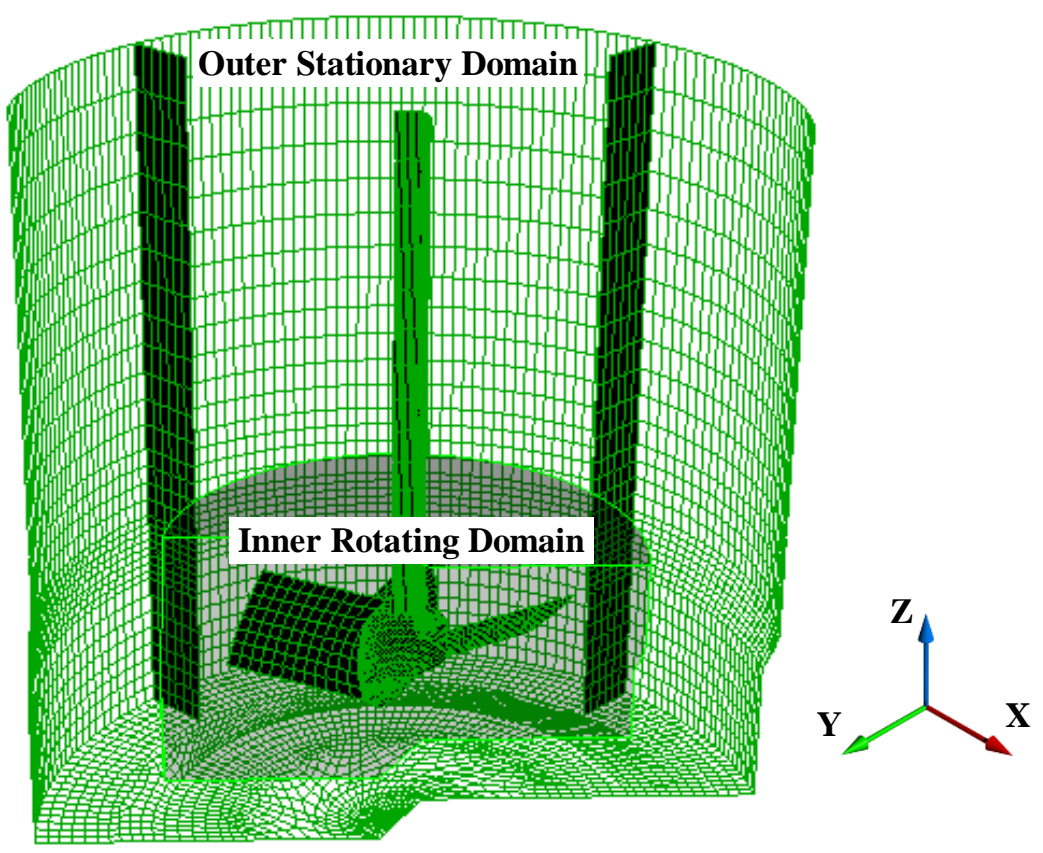

Figure 3. Computational domain and grid distribution in stirred tank

\section{Numerical simulations}

The stirred tank consists of 6 blades and 4 baffles and, therefore can be divided into two symmetrical parts. Only concern is the width of the blade and diameter of hub is $0.034 \mathrm{~m}$, which results in the overlapping of blades near the hub. Despite the overlap, the symmetry is still preserved and half of the tank can be simulated 
by considering rotationally periodic nature of geometry. To eliminate any possibility of error due to considering half of the tank, simulation of full scale tank is conducted and its results are compared with rotationally periodic half geometry.

For simulating rotation of impeller blades sliding mesh, Inner-Outer (IO) or Multiple Reference Frame (MRF), Sliding Grid Approach (SG) and Large Eddy Simulations (LES) models can be used. While MRF provides results with reasonable accuracy, the results of SG are slightly accurate compared to this approach (Aubin et al., 2004; G. Montante \& Magelli, 2005). However, the SG approach requires an order of magnitude higher computational power for the marginal gain in accuracy (Ranade, 2001). Compared to these approaches, the computational power required by LES is two order of magnitudes higher than that required by the former two approaches (J. Derksen et al., 1999). Therefore, for the current study, MRF approach is used to simulate impeller rotation. A reference moving zone with dimensions $r=0.06 \mathrm{~m}$ and $0.036<\mathrm{z}<0.137$ was created (where $\mathrm{z}$ is the axial distance from the bottom). The impeller rod outside this zone is considered as a moving wall. Impellers used in all the cases simulated in the study are operated in the down-pumping mode. The top of the tank is open, so it is defined as a wall of zero shear. It is to be noted that only the steady state results at just suspension speeds are used in the study, thus the impact of initial conditions of uniform concentration is negligible on the results. Sardeshpande et al. (2010) also suggested that the initial condition of uniform concentration has no influence on the final steady state results at very low impeller speeds and speeds at or around the just suspension speed. Therefore, a uniform average concentration of particles in the tank as an initial condition of the simulation is taken. For modelling the turbulence, a standard $k-\varepsilon$ mixture model is used. The model parameters are $\mathrm{C} \mu: 0.09, \mathrm{C}_{1}: 1.44, \mathrm{C}_{2}: 1.92, \sigma_{\mathrm{k}}: 1.0$ and $\sigma_{\varepsilon}=1.3$. In the present work, SIMPLE scheme is used for Pressure-Velocity coupling along with the standard pressure interpolation scheme. To avoid any numerical diffusion and unphysical oscillations, a third order Quadratic Upstream Interpolation for Convective Kinetics (QUICK) discretization scheme is used for momentum, volume fraction, turbulent kinetic energy and turbulent dissipation rates. The convergence of the simulation is verified by monitoring residual values as well as additional parameters namely turbulence dissipation over the volume, turbulence dissipation at the surface right below impeller and torque on the shaft. Once the residuals and additional parameters are constant, a simulation is deemed to be converged. The steady-state simulations resulted in the strong variation in the solid volume fraction in the stirred tanks, and to maintain the mass of solids constant, the numerical strategy is changed and a pseudo-transient or false time stepping approach is used. The time step used in the simulations is $0.0001 \mathrm{~s}$, which is gradually increased to $0.001 \mathrm{~s}$. The simulations took $10 \mathrm{~s}$ to attain steady values, and after $10 \mathrm{~s}$ no change in the local values of any local parameter is observed. However, to eliminate any possibility of minor fluctuations in the simulation results, the simulations are conducted for $15 \mathrm{~s}$ and the data is time averaged for the last $3 \mathrm{~s}$. The numerical solution of the system is obtained by using the commercial CFD solver ANSYS FLUENT 15.0 on 6 cores, 12 thread Intel ${ }^{\circledR}$ Xeon $^{\circledR} 3.2$ 
$\mathrm{GHz}$ processor workstation with 64 bit operating system. Each time step takes an average of 1 minute of the wall clock time.

The simulation domain is discretised using a mesh that can resolve the physics inside the stirred tank with accuracy. Refining the mesh incurs computational power and time and therefore, a mesh needs to be finalised so that the simulation results do not alter with further refining the mesh. For the purpose, a grid independency test is conducted initially on the single phase flow and is presented. Extensive validation of models used for simulating high solid loading stirred systems is not published in the literature and therefore, the models used in the paper are validated with the experimental results upto $40 \mathrm{wt}$. $\%$ of solid concentration in stirred tanks of Guida et al. (2010). The turbulence models for the single phase simulations of stirred tanks are extensively assessed by Murthy and Joshi (2008). The turbulence characteristics in the stirred tanks are expected to change in the presence of particles and consequently, the inferences drawn from the study might not be applicable. Therefore, to capture the influence of different turbulence models on the prediction, the models (EE or EEKTGF, Drag force and turbulent dispersion forces) for the precisely simulating the suspension of secondary phase (solids) are finalised first, and later on, various turbulence models and their impact on the predictions are examined. In this direction, a base-case is chosen in which the simulations are conducted using EE and EE-KTGF approach, where both of the results using both the simulation techniques are compared. For EEKTGF, the default value of 0.9 for the restitution coefficient has been used, which is varied later to understand its influence on the simulation results. The drag and turbulence model used in the base-case are HuilinGidaspow model, that takes the dense particle effect into account along with a smooth shift from Wen-Yu to Ergun model, and the most widely used k- $\varepsilon$ turbulence mixture model. Dispersion force is neglected in the initial simulations. The models are examined in the order of modelling approaches, coefficient of restitution, interphase drag force, turbulent dispersion force and turbulence models. After each comparison, the parameters from the base-case are changed based on the most appropriate model finalised from the investigation.

\section{RESULTS AND DISCUSSION}

\section{Grid Independency, Geometric Periodicity and Flow Field}

Grid independency is important for gaining reliable results, therefore a grid independence study was conducted before using the grid for further simulations. The experimental values for axial, tangential and radial velocities at impeller discharge plane for single phase flow were compared with the simulation results using computational grids with 65000, 480000 and 1600000 cells (see Figure 4a). The mesh with 65000 cells provides results with reasonable accuracy. However, for accurate results near the wall, a refined mesh is required to resolve the $\mathrm{y}^{+}$values, which is obtained in the geometry of 480000 cells. No considerable change in the simulation predictions is visible by further refining the mesh. This mesh is further used to test whether applying the rotational periodicity in the current geometry is influencing the simulation results. For the 
comparison, a full geometry is also constructed with 960000 cells and both of these geometries are simulated using identical single phase conditions. The radial, axial and tangential velocities at the impeller plane obtained using both of these simulations are plotted in Figure 4 (b). The results from both the cases coincide for all the three velocity components, and therefore it can be concluded that the rotationally periodic geometry is equally accurate for simulating the flow in stirred tanks. Hence, the mesh with 480000 cells in half rotationally periodic geometry is used for the rest of the simulations in the study.

The axial, tangential and radial velocity plots describe the flow generated by the PBTD. The PBTD pumps the fluid downwards leading to highly negative axial velocities at the impeller plane. The jet leaving the impeller flows down to the bottom of the stirred tank, is redirected towards the periphery and circulates back to the top along the walls. Due to such motion, a flow loop is formed near the impeller. It results in the decreasing axial velocity when moving radially outwards in impeller plane that eventually increases due to the upwards flow near the walls. $45^{\circ}$ inclination of the impeller blade imparts momentum in the tangential direction resulting in moderate values of tangential velocity. Due to the downward flow developed by a PBTD, magnitude of the radial component of velocity is the lowest. The maximum values of radial, tangential and axial velocity in the impeller plane are $0.1 \mathrm{U}_{\text {tip }}, 0.28 \mathrm{U}_{\text {tip }}$ and $0.45 \mathrm{U}_{\text {tip }}$ respectively. Both radial and tangential components gain value with the increase in radius as the velocity increases radially along the impeller blades. The maximum value is attained by both of these components close to impeller tip after which a sudden decline is observed due to no momentum source in the absence of impeller. These characteristics are well represented in the velocity profiles shown in Figure 4.

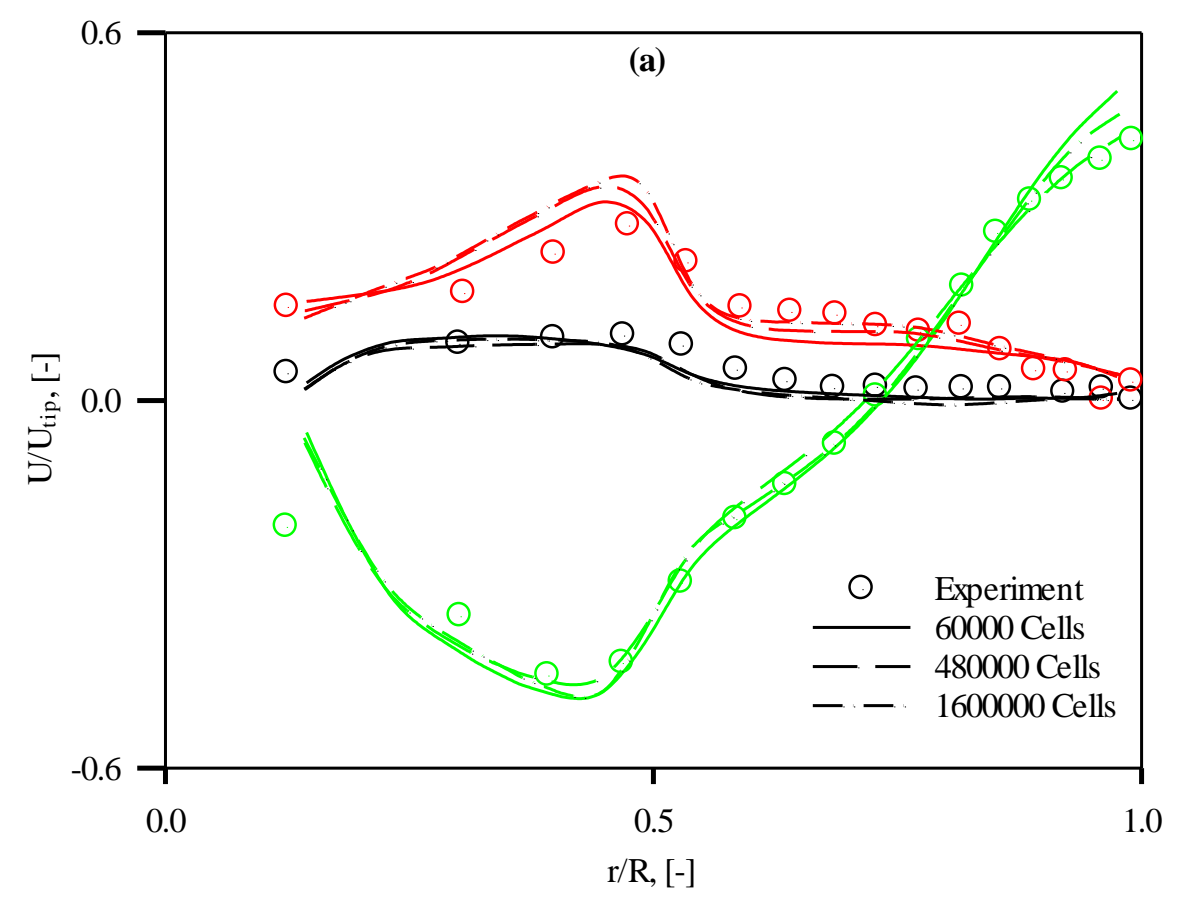




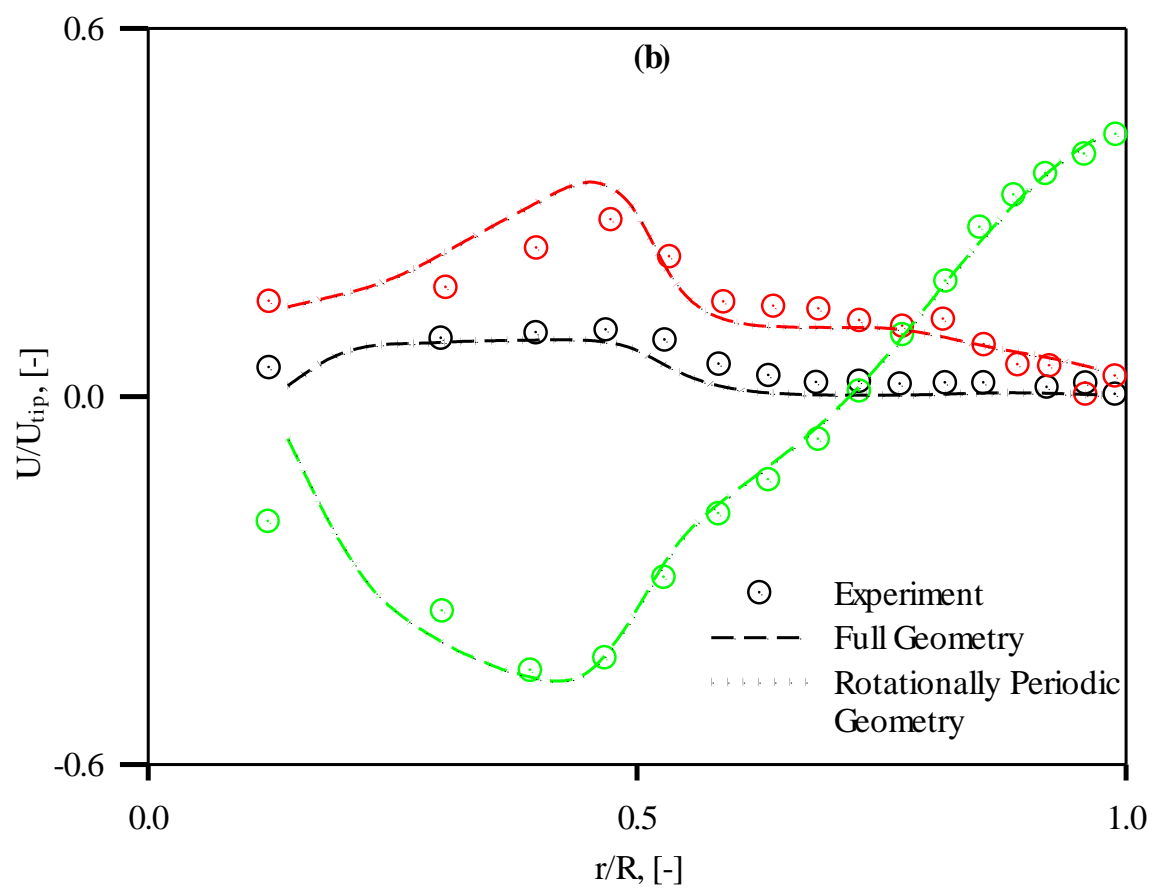

Figure 4. Comparison of dimensionless axial (green), radial (black) and tangential (red) velocity at $\mathrm{z}=0.3 \mathrm{H}$ plane for (a) computational grid with different resolution and (b) Full and rotationally periodic geometry.

\section{EE and EE-KTGF Approach}

For the initial simulations, Huilin-Gidaspow drag model was used and simulations were conducted with EE approach and EE-KTGF approach to evaluate their applicability in the cases. In both the approaches, the liquid and solid phases are treated as fluids in interpenetrating fluids for which the continuity equations are solved. Only exception is that the particle-particle interactions are taken into account in the momentum equation in EE-KTGF method. Solid pressure, radial distribution, frictional force, etc. are implemented indirectly in the momentum equations. The closure for the stress term of the particle phase is provided using kinetic theory for granular flow in the case. In the theory, the behaviour of particle motion is based on the analogy to the kinetic theory of dense gases. As is the case for gases, the collisions of particles being swept along by the mean flow are assumed as a result of the particle velocity fluctuations about the mean. The intensity of the particle velocity fluctuations determines the stresses, viscosity, and pressure of the solid phase. In the EE method, the equations used are the same as that of fluid that utilizes Boussinesq's eddy viscosity hypothesis for the closure of momentum equation. It only takes into account fluid-solid interactions in the form of drag and has no term to account for particle-particle interaction.

Figure 5 shows the comparison of flow field generated in half of the stirred tank in the presence of solids between experiments and the two computational approaches. In all of these cases, the qualitative nature of the jet is similar to that of single phase flow. The exception can be observed at high solid loading at which the jet diverts well before hitting the bottom of the stirred tank. A high velocity magnitude is observed in the impeller region and along the jet, but low velocity is noticed at the center of loop (eye) and region near top of tank. 
Similar behaviour was observed with the studies that used axial impellers for the investigations (Bittorf \& Kresta, 2003; Guida et al., 2010). At low solid concentrations, the flow field predicted using EE approach and EE-KTGF approach do not differ. In such conditions, both approaches present flow field with reasonable accuracy. However, it is worth noticing that no visible change in the flow field is observed in the stirred tank with increase in solid concentration using EE simulations. The predicted behavior of the jet remains unaltered with the increasing concentration of solids and the jet maintains its high inclination and velocity magnitude. It causes the surrounding fluid to flow due to shear and result in over-prediction of velocity near the bottom centre. In such scenario, it changes the direction only when it strikes the bottom of the tank. In contrast to this, experimental results show dampening of velocity field in the impeller discharge region with the increase in solid concentration. Furthermore, the jet changes its direction before it contacts bottom at high solid loading in the presence of solids as the turbulence around and below the impeller increases significantly. EE-KTGF simulations are able to predict this behavior while taking into account the energy dissipated by particle-particle interaction. The maximum value of turbulence kinetic energy at impeller plane as predicted by EE-KTGF simulation is $8.093 \mathrm{~m}^{2} / \mathrm{s}^{2}$ while the same in EE simulations is $1.12 \mathrm{~m}^{2} / \mathrm{s}^{2}$. With the high value of turbulence kinetic energy and smaller flow loop, the EE-KTGF simulations are able to simulate the flow behaviour highlighted by Guida et al. (2010) at high solid concentration. This approach also predicts the qualitative and quantitative values of flow with reasonable accuracy. Kasat et al. (2008) and Sardeshpande, Sagi, Juvekar, and Ranade (2009) pointed out that in case of high solid concentration, solid accumulated at the bottom offers resistance to the flow loop and termed it as "false bottom effect". This decreases the velocities in the vicinity of the bottom of the tank. The diminution of velocity was also indicated by Guida et al. (2010) and is also observed in the simulation results using EE-KTGF approach. This approach is also able to simulate a smaller jet loop formed near impeller with an elevated eye of jet, which is not the case in EE simulation results. 


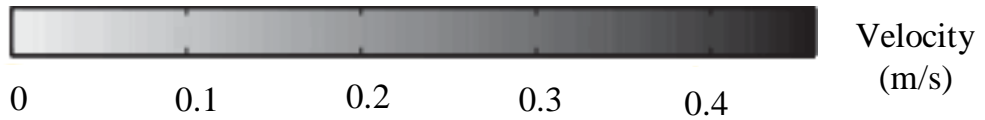

(a)

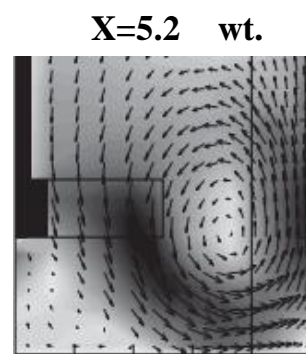

$\mathrm{X}=\mathbf{1 0 . 6}$ wt.

$\mathrm{X}=20$ wt. $\%$
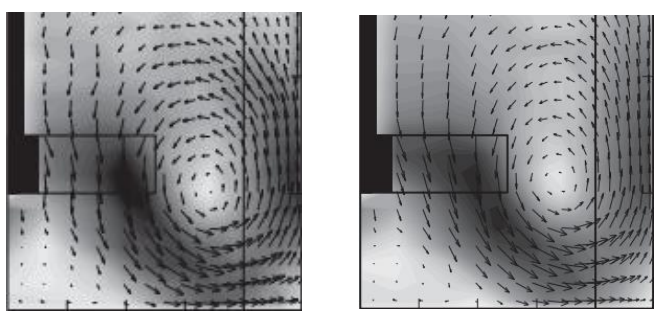

$\mathrm{X}=\mathbf{4 0}$ wt. $\%$

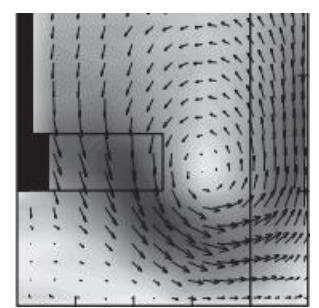

(b)
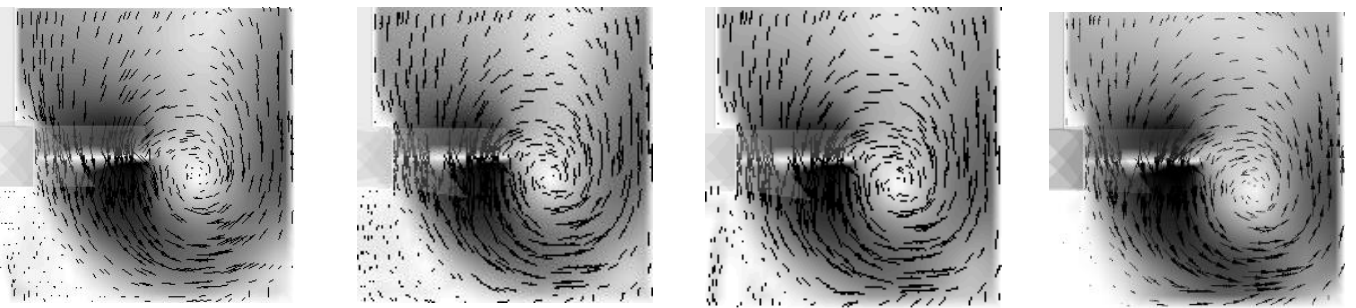

(c)
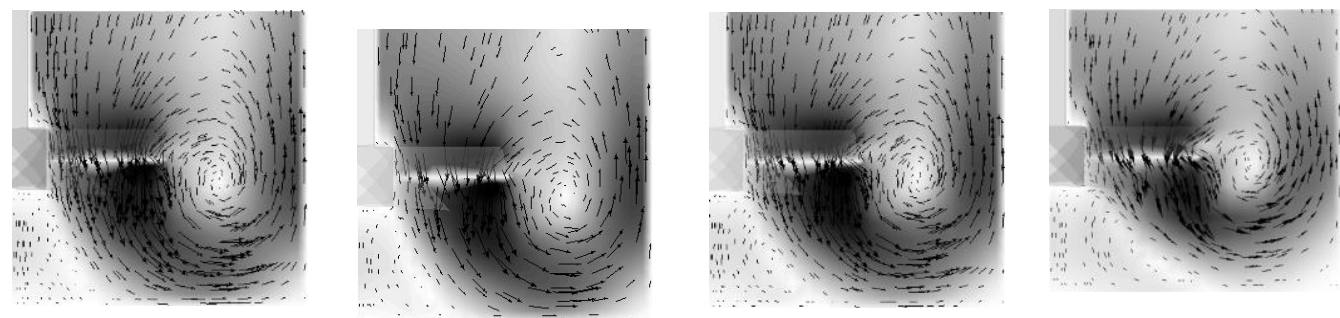

Figure 5. Flow field around and below the impeller shown as velocity vectors and velocity profiles of radial-axial velocity using (a) experimental data, (b) EE approach and (c) EE-KTGF approach.

The radially averaged normalised axial concentration profiles from experiments are compared with the predictions using both the simulation approaches are shown in Figure 6. Although the simulation results show reasonable agreement with the experimental data, these plots are required to be analysed in conjunction with the radial concentration profiles. The results presented in Figure 6 are averaged azimuthally over an axial plane and may not reveal a disparity if simulations over-predict and under-predict at different radial positions in the same plane. Therefore, the radial profiles at various heights are also presented in Figure 7. 


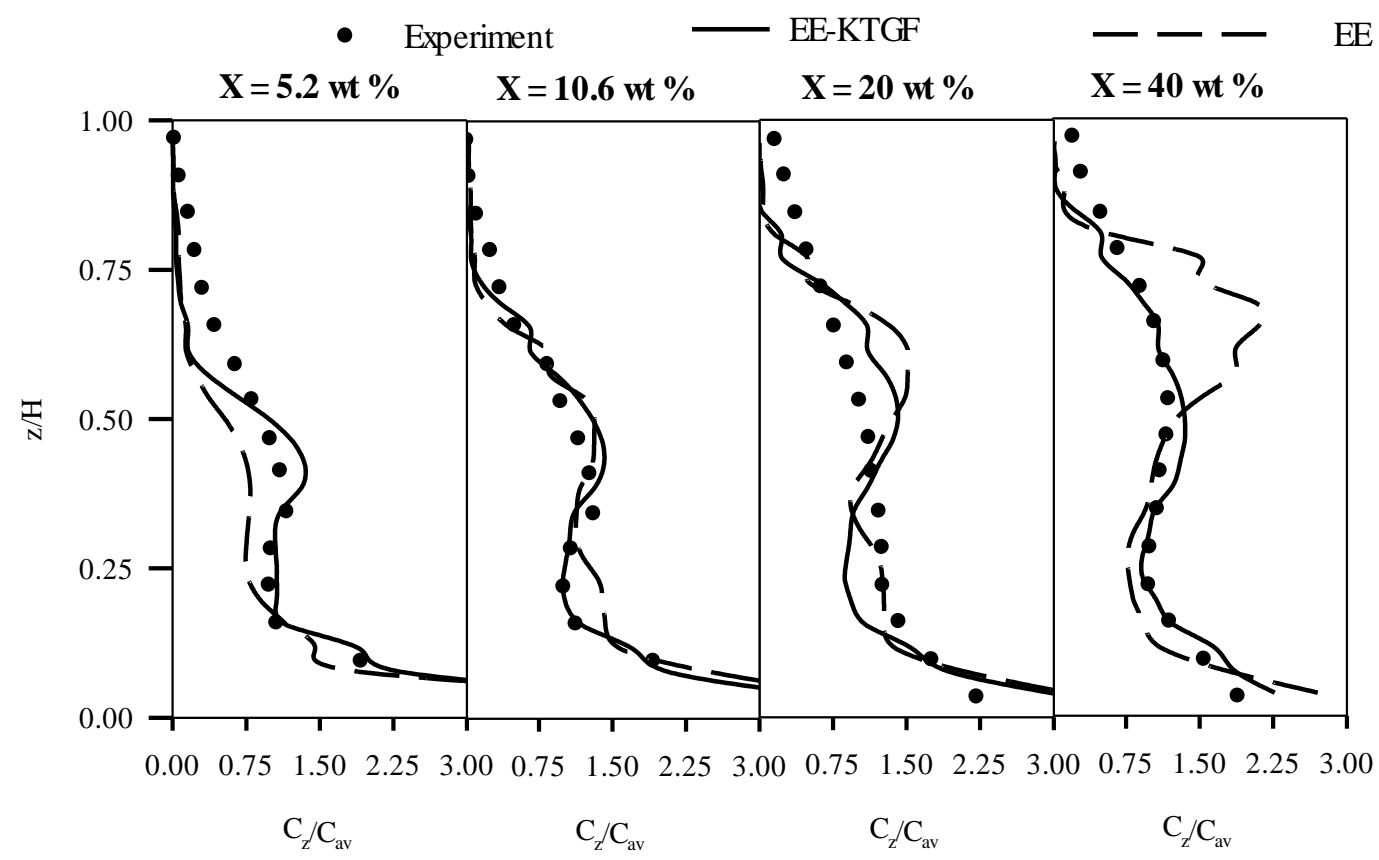

Figure 6. Azimuthally and radially averaged normalized solid concentrations plotted axially at $\mathrm{N}=\mathrm{Njs}$.

In low solid concentration cases (5.2 wt.\% and $10.4 \mathrm{wt} . \%$ ), both EE and EE-KTGF approach appear to provide results with reasonable accuracy as shown in Figure 6. On examining the radial concentration profiles in Figure 7, it appears that the latter approach grossly over-predicts the solid concentration at the bottom centre of the tank. This highest disparity is due to accumulation of solid in the dead zone formed in this region. For planes at height of $0.29 \mathrm{Z}$ and $0.43 \mathrm{Z}$, the over-prediction suggests the high concentration of solids in the lower half of the tank, which also resulted in the under-prediction of solid concentration by the EE-KTGF approach above the dimensionless height of 0.5 (see Figure 6). A closer look at the turbulent kinetic energy values for both the cases reveal that the turbulence is dampened in the EE-KTGF approach and its values are reduced by $50 \%$, thus hindering the suspension. The maximum value of turbulent kinetic energy for EE and EE-KTGF cases at 5.2 wt. $\%$ solid content are $1.075 \mathrm{~m}^{2} / \mathrm{s}^{2}$ and $0.5474 \mathrm{~m}^{2} / \mathrm{s}^{2}$ respectively. Therefore, the solids are suspended in the high turbulence regions around impeller and not beyond that. As the height increases, a sudden drop in the turbulence kinetic energy results in decreased suspension, which is significant in EE-KTGF cases. EE approach faces the same problem at very high $\mathrm{z} / \mathrm{Z}$ values of 0.71 and above due to the lower values of turbulence in these regions. Moreover, the granular temperature attains moderate values around impeller, which are not sufficient for the suspension of particles. It is not considered in the EE approach and therefore, the suspension is accelerated by the higher values of isotropic turbulence. 


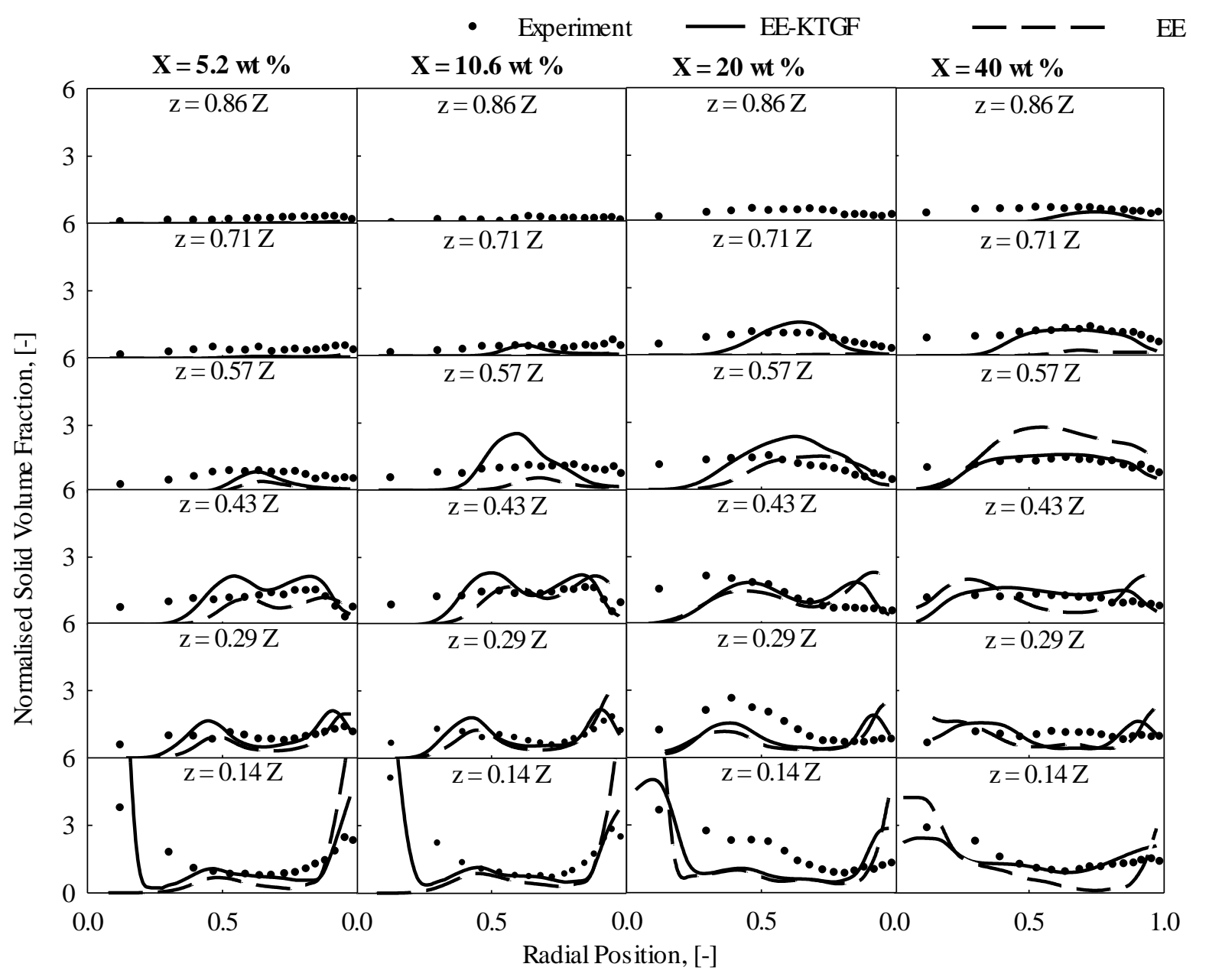

Figure 7. Circumferentially averaged normalized radial concentrations profiles at various heights at $\mathrm{N}=\mathrm{Njs}$.

With the increasing solid concentration, the EE approach becomes increasingly inefficient in the prediction of solid concentration profiles. At high solid concentrations (20 wt.\% and $40 \mathrm{wt} . \%)$, it is evident from Figure 6 that EE-KTGF approach more accurately predicts the solid concentration in high solid loading stirred tanks. It suggests that at high concentration, the contribution of turbulent kinetic energy (or granular temperature) of the particles plays a significant role. The value is expected to be high due to the increased collisional energy as a result of interaction of particles. It is not taken into account in the EE approach and has led to the discrepancy in the solid concentration predictions. The highest deviation in the EE simulation prediction is observed in the upper half region of the stirred tank where the solids are dragged along the circular loop formed by the impeller rotation. The solid tend to accumulate in the low to moderate velocity zones below the impeller in the stirred tank. Due to high mean velocity and bigger radius of the loop, the particle concentration in the radius of loop beneath the impeller is less than $5 \%$. As the intensity of jet formed by the impeller is not changed in EE simulations, the solid concentration remains low at the point where it hits the bottom. The solid is dragged along with the flow towards the bottom-periphery of the tank and is accumulated (see Figure 7). Similar accumulation in the low-velocity zones at the bottom-centre is also observed. Some solid also get drifted along the flow above the impeller and result in over-prediction of volume fraction. The value of turbulent kinetic energy in the region around and below the impeller is crucial as its predictions are highly 
influenced by the solids present in the region. The turbulent kinetic energy increases in the presence of particles with large diameter as the wakes behind the particles result in generation of turbulence (Crowe, 2000). High concentration above the impeller also leads to the higher values of turbulent kinetic energy in this region compared to the bulk region. In the EE-KTGF approach, the particle phase turbulence along with the solid pressure is modelled correctly that has led to the accurate prediction of flow field and hence the solid volume fraction profile.

It is evident from the above discussion that the predictions from the EE-KTGF approach are better than the EE approach. Therefore, now onwards EE-KTGF modelling approach will be used for the further investigation of drag force, turbulent dispersion force and turbulence models. The EE-KTGF uses the calculation of granular temperature to incorporate the properties of particles in the momentum equation through solid pressure and solid shear stresses. EE-KTGF approach considers particle collisions using coefficient of restitution, and the effect of its value is examined on the numerical predictions before other investigating models.

\section{Coefficient of restitution}

Increase in the coefficient of restitution increases the elasticity of particle-particle collision, which has reflected in the increased uniformity of distribution from $82.37 \%$ to $85.7 \%$. The value of restitution coefficient suggests the amount of energy conserved during the particle-particle collisions. Restitution coefficient of 0.99 means that nearly all the energy is conserved in the process. Therefore, it leads to an increased scattering of the particles, which result in increased suspension at the restitution coefficient of 0.99. The suspension in the cases is calculated using the criterion proposed by Tamburini et al. (2011). According to this criterion, the solids present at the bottom of the tank with volume fraction greater than or equal to the packing limit are considered as unsuspended solids. Therefore, the percent suspension can be expressed as:

$$
\mathrm{S}=\left(1-\frac{\mathrm{V}_{\text {unsuspended }}}{\mathrm{V}_{\text {total }}}\right) \times 100 \%
$$

The particles are completely suspended, if the value of $S$ reaches 1 and the suspension decreases with the degradation of its value. With the increased coefficient of restitution from 0.9 to 0.99 , this value increased from $98.2 \%$ to $99.4 \%$. It resulted in the accurate prediction of solid concentration at the bottom of the tank. However, it has adversely affected the results in the impeller plane of the stirred tank, where solid concentration is over-predicted by the use of high value of restitution coefficient $(=0.99)$. As can be seen in Figure 8 and Figure 9, the increase in restitution coefficient has decreased the solid accumulation at the bottom of the tank. The conserved energy in the process with high value of restitution coefficient is reflected in more dispersed solids at the bottom centre. The coefficient of restitution is likely to be lower for dense regions as the collisions of the particles become less ideal (Goldschmidt, Kuipers, \& van Swaaij, 2001). Figure 8 (a) supports this fact and has shown denser solids at the bottom of the tank when compared with other cases. 
(a)

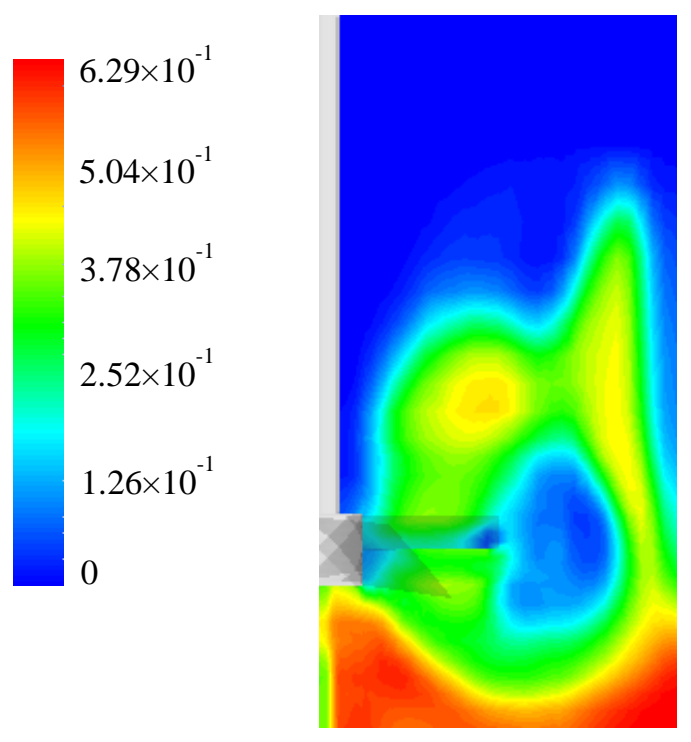

(b)

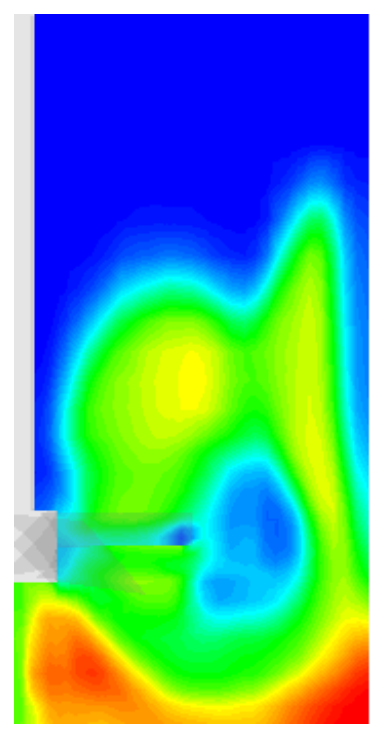

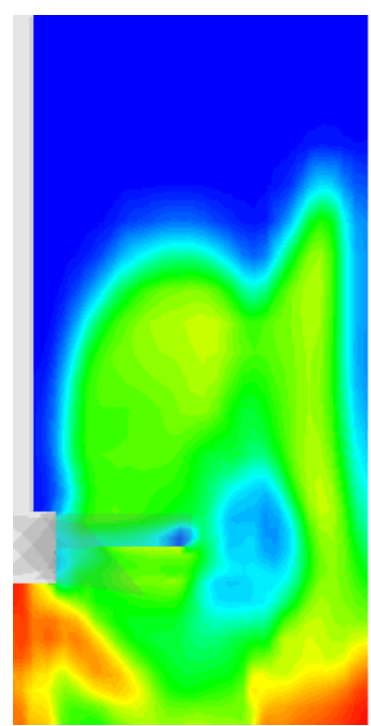

Figure 8. Solid volumetric concentration contours for $40 \mathrm{wt} \%$ case at mid-impeller plane with restitution coefficient of (a) 0.9 , (b) 0.95 and (c) 0.99 .

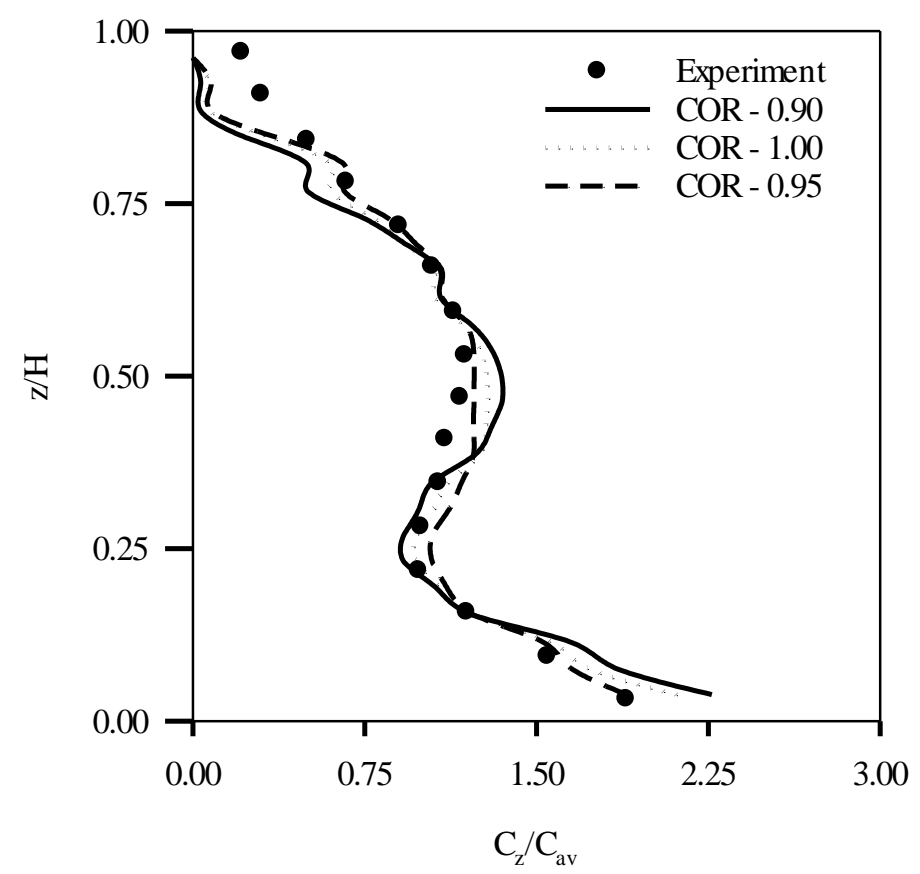

Figure 9. Comparison of coefficient of restitution (COR) using normalised axial concentration profiles at $\mathrm{N}=\mathrm{N}_{\mathrm{js}}$ for $40 \mathrm{wt}$. $\%$ case.

Similar characteristics are reflected in the average solid concentration plotted in Figure 9, with lower concentration at the bottom with increasing restitution coefficient. The mean volume fraction has increased at the impeller plane due to increased suspension. But in the COR-0.99 case, the solid concentration is overpredicted due to increased suspension. The effect of coefficient of restitution is also dominant in the region above the impeller, where the zone of high solid concentration diminishes with increased value of coefficient of restitution. It, therefore, facilitates to increase the accuracy of prediction in this zone, where the simulations over-predict the average solid concentration values. Disparity in the solid concentration predictions near the 
top of the stirred tank cannot be addressed by coefficient of restitution as the solid concentration remains negligible even with the use of its highest value. It is evident from this discussion that the variation of coefficient of restitution has a direct and prominent impact on the solid distribution and therefore, its value is critical for the suspension prediction in high solid loading stirred tanks.

From the comparison of the experimental data, the intermediate value of 0.95 for restitution coefficient appears to represent the system more closely compared to 0.9 and 0.99 , and therefore, this value will be used in the rest of the study.

\section{Interphase Drag Force}

In the current cases, the influence of coarse particles on the predictions of modified Brucato drag model is evident from Figure 10. Specifically at the lower concentrations, the predictions are eccentric as the particles diameter to Kolmogorov length ratio is high and yields a very high value of drag. When seen in conjunction with Figure 2, the difference in the predicted drag force elevates with lowering volume fraction. Same is reflected in the cases, where the predictions improve with increasing solid loading. Modified Brucato drag model modifies the drag according to the dominating turbulence below the impeller. The average value of the Kolmogorov length scale is of the order of $10^{-5}$. Therefore, the increase in the drag is severely over-predicted. As compared to below impeller zone, the turbulence dissipation rates in this region decrease by an order of magnitude which renders a minute change in the value of drag exerted. With an over-predicted value of drag in this region, presence of a high solid concentration is evident. Radial profiles shown in Figure 11 suggest that the high magnitude of drag force drives the solids off the impeller region and the solids suspend in the middle region of the stirred tank. Therefore, the under-prediction in the solid concentrations below $0.43 \mathrm{z}$ height is also accompanied by significant overshoot of this quantity around the height of $0.57 \mathrm{z}$. The influence is gradually dampened with concentration increase to $20 \mathrm{wt} \%$ and $40 \mathrm{wt} \%$, where the error reduces substantially. Above $0.57 \mathrm{z}$, the overshoot continues further for $5.2 \mathrm{wt} \%$ and $10.6 \mathrm{wt} \%$ cases, while it diminishes quickly for higher concentrations. For higher solid concentrations, the value of Kolmogorov length in the top zone of stirred tank increases sharply with the decrease in the dissipation rate. Its order of magnitude approaches that of particle diameter, resulting in the minimum modification of the drag force. In this case, the drag is equal to Wen-Yu drag and only dependent on the local volume fraction.

Therefore, it is clear from the above discussion that the correction factor in Brucato and modified Brucato drag law is not applicable in the current conditions. The variation of the drag force with the ratio of particle diameter to the Kolmogorov length scale provides unrealistic results for coarse particles. The factor implemented in these correlations may be a lumped parameter that needs to be investigated by assessing the influence of other forces such as turbulent dispersion force and turbulent kinetic energy. Their applicability is so far limited to particle diameters lower than $600 \mu \mathrm{m}$. 
- Experiment $\longrightarrow$ H-G $\ldots . . . \cdot$ M-B $\quad--$ Syamlal $-\cdots-\cdots-\cdots$ Tenneti

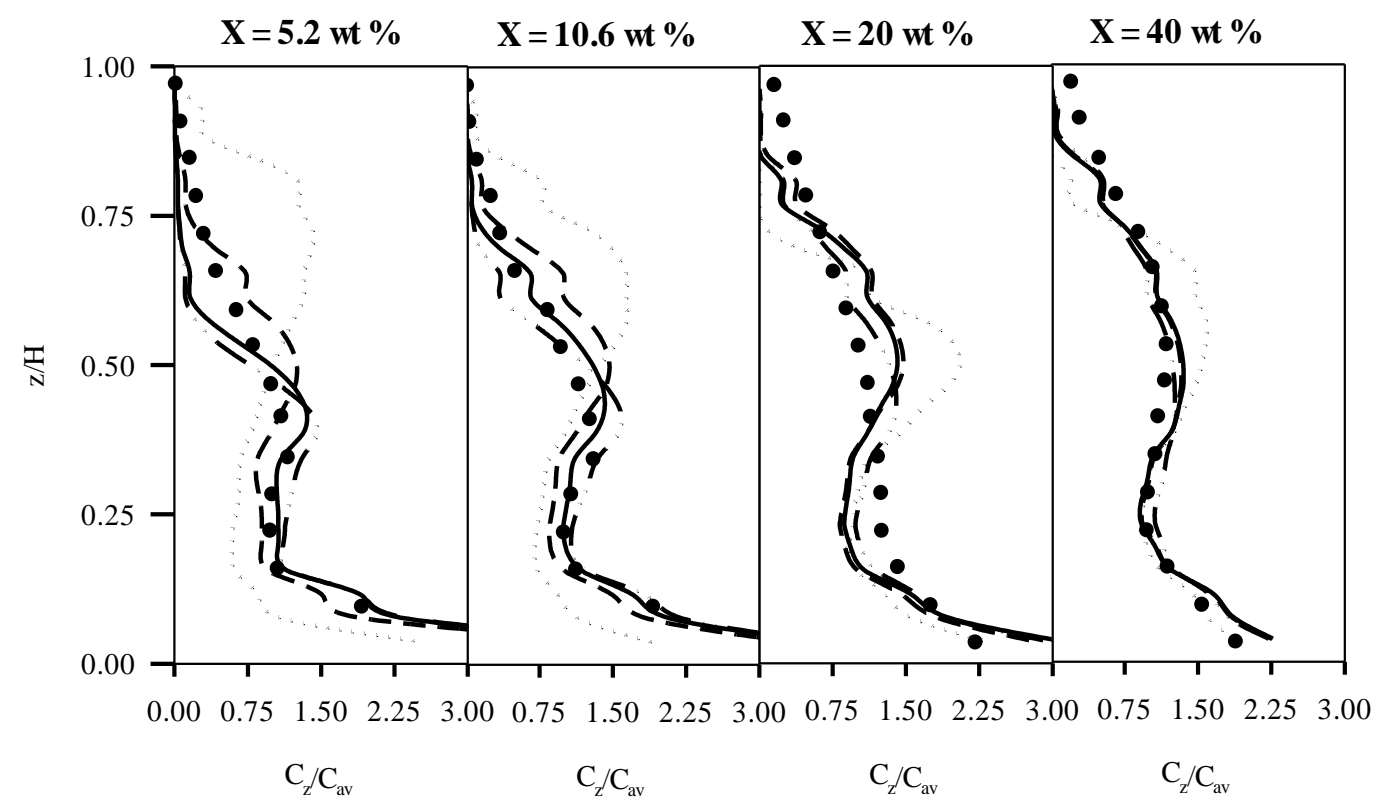

Figure 10. Azimuthally and radially averaged normalized solid concentrations plotted axially at $\mathrm{N}=\mathrm{Njs}$.

Huilin-Gidaspow drag model takes into account different correlations for dilute phase (Wen \& Yu, 1966) and dense phase (Ergun, 1952), and provides a smooth switching between the shift (Huilin et al., 2003). In the region around and below the impeller for average solid loading below $10.6 \%$, this model is able to predict the solid concentration profiles with reasonable accuracy. In these cases, the model takes the form of Wen-Yu model as the solid volume fraction remains below $20 \%$ in approximately $99 \%$ of tank volume. In such conditions, the values of Huilin-Gidaspow model approaches Tenneti drag model and both of these have similar predictions. At higher solid loadings, Huilin-Gidaspow deviates from Tenneti drag and approaches Syamlal \& Brien drag model. It is reflected in Figure 10 and Figure 11 where the predictions of HuilinGidaspow drag are similar to Tenneti drag at low solid loading and are similar to Syamlal \& Brien drag at high solid loading. At high solid loading, the liquid volume fraction in the region around and below the impeller is less than 0.8 and therefore any increase in drag in these regions are taken into account by Ergun equation which is based on solid concentration. The values from Tenneti drag are lower than Huilin-Gidaspow, which renders lower suspension. As a result, much lower average volume fraction of solids is evident at height of $0.43 \mathrm{z}$ or higher. This effect is specifically highlighted at low solid loadings due to accumulation of solids at the bottom and unavailability of solids in the upper region of tanks. At $40 \mathrm{wt} \%$, predictions of HuilinGidaspow, Tenneti and Syamlal \& Brien drag model are in reasonable agreement with the experimental data. Tenneti drag differs from the other two, but the difference is negligible.

Considering the axial concentration profiles, it appears that Syamlal drag model is applicable for low, moderate and dense solid loading stirred tanks systems. However, in making such a statement, radial concentration profiles cannot be neglected. Syamlal drag model maintains its efficiency at higher solid loading 
upto a height of $0.57 \mathrm{z}$, above which it under-predicts the solid concentration values. At low solid loading systems, the predictions compare well for height below $0.43 \mathrm{z}$, above which an overshoot is apparent at $0.6 \mathrm{R}$. It is also worth noticing that for all the drag models, the efficacy of predictions is considerably reduced near the top of the tanks. However, comparing with the other models, the error in predictions using Syamlal drag model is minimal for the simulation of solid loading stirred tanks over the range of solid volume fraction studied in the paper. In the remaining study presented in this paper, Syamlal drag model is used to analyse other governing factors contributing to the complex hydrodynamics in stirred tanks.

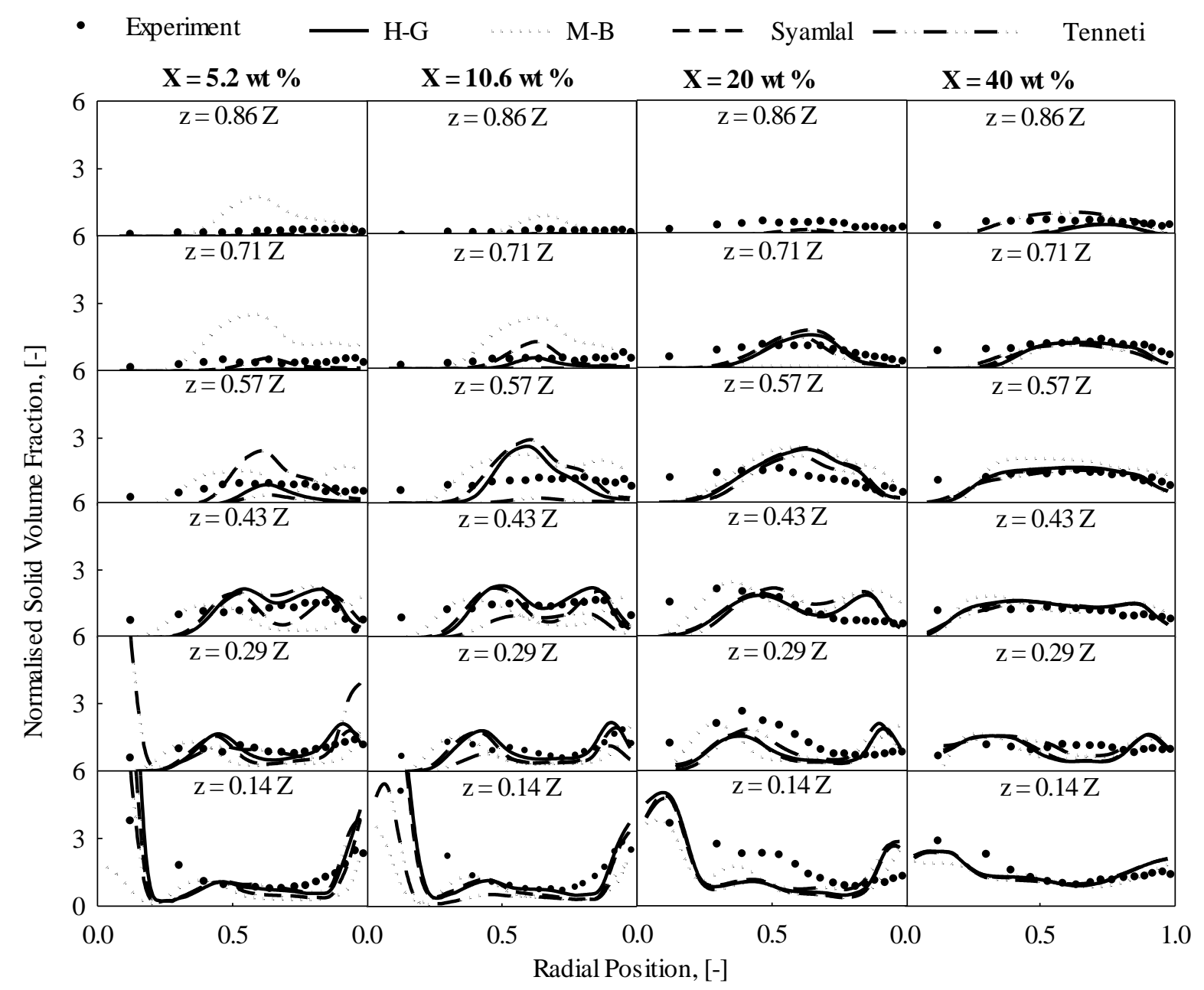

Figure 11. Circumferentially averaged normalized radial concentrations profiles at various heights at $\mathrm{N}=\mathrm{Njs}$.

\section{Turbulent Dispersion Force}

de Bertodano et al. (1994) (now onwards referred to as DBL), Simonin and Viollet (1990), and Burns et al. (2004) models are present to model the turbulence dispersion force in Fluent. Both Simonin et al. and Burns et al. models calculate the force by evaluating the drift velocity. The difference between the two is that the latter derives the value of dispersion scalar from the kinematic viscosity of the continuous phase, while the former uses the mixture turbulent kinematic viscosity. DBL, on the other hand, uses the gradient of dispersed phase volume fraction rather than drift velocity to calculate the turbulent dispersion force. 


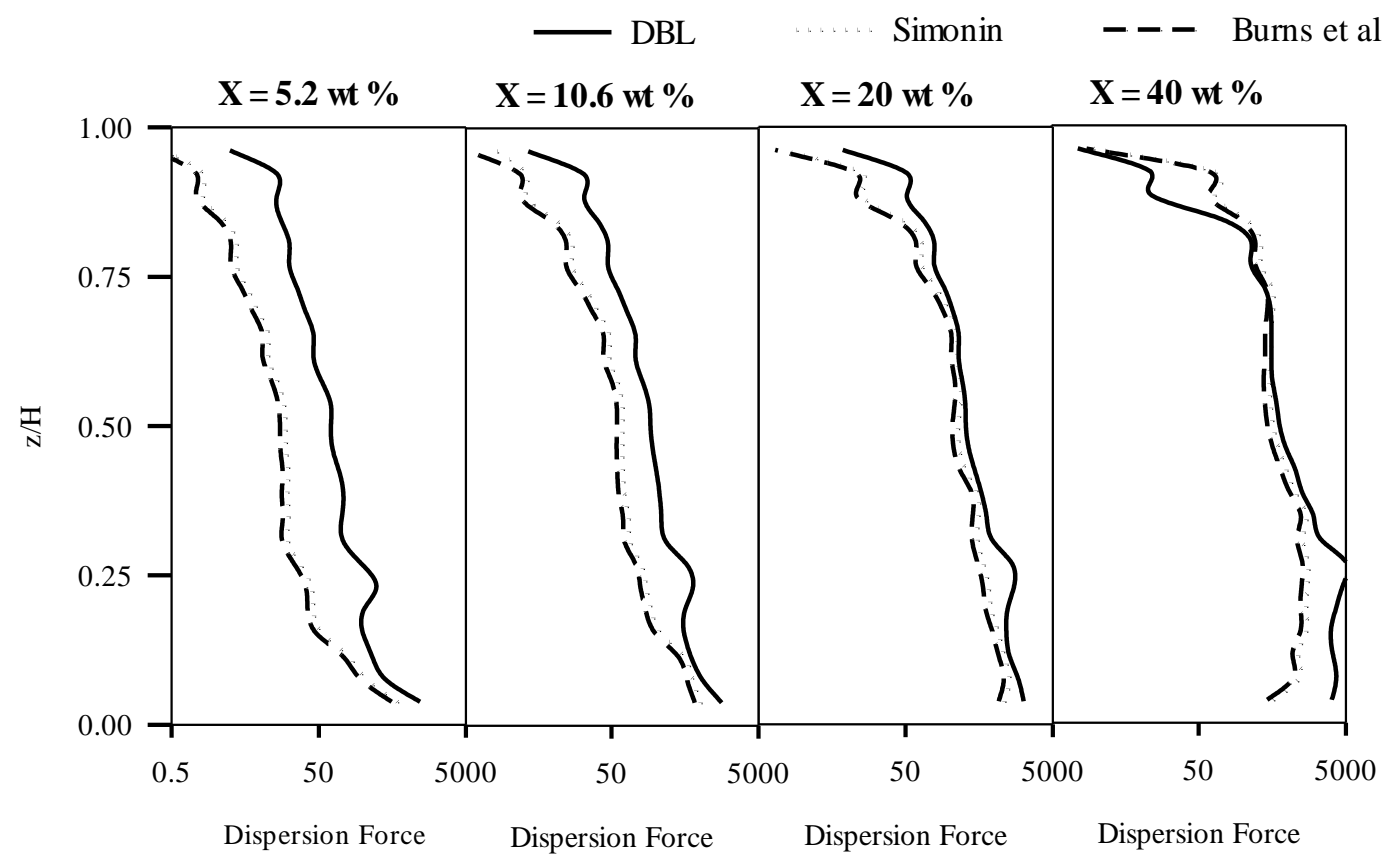

Figure 12 . Azimuthally and radially averaged dispersion force plotted axially at $\mathrm{N}=\mathrm{Njs}$.

- Experiment No Dispersion DBL $\quad--$ Simonin $-\cdots-\cdots-\cdots$ Burns et al.

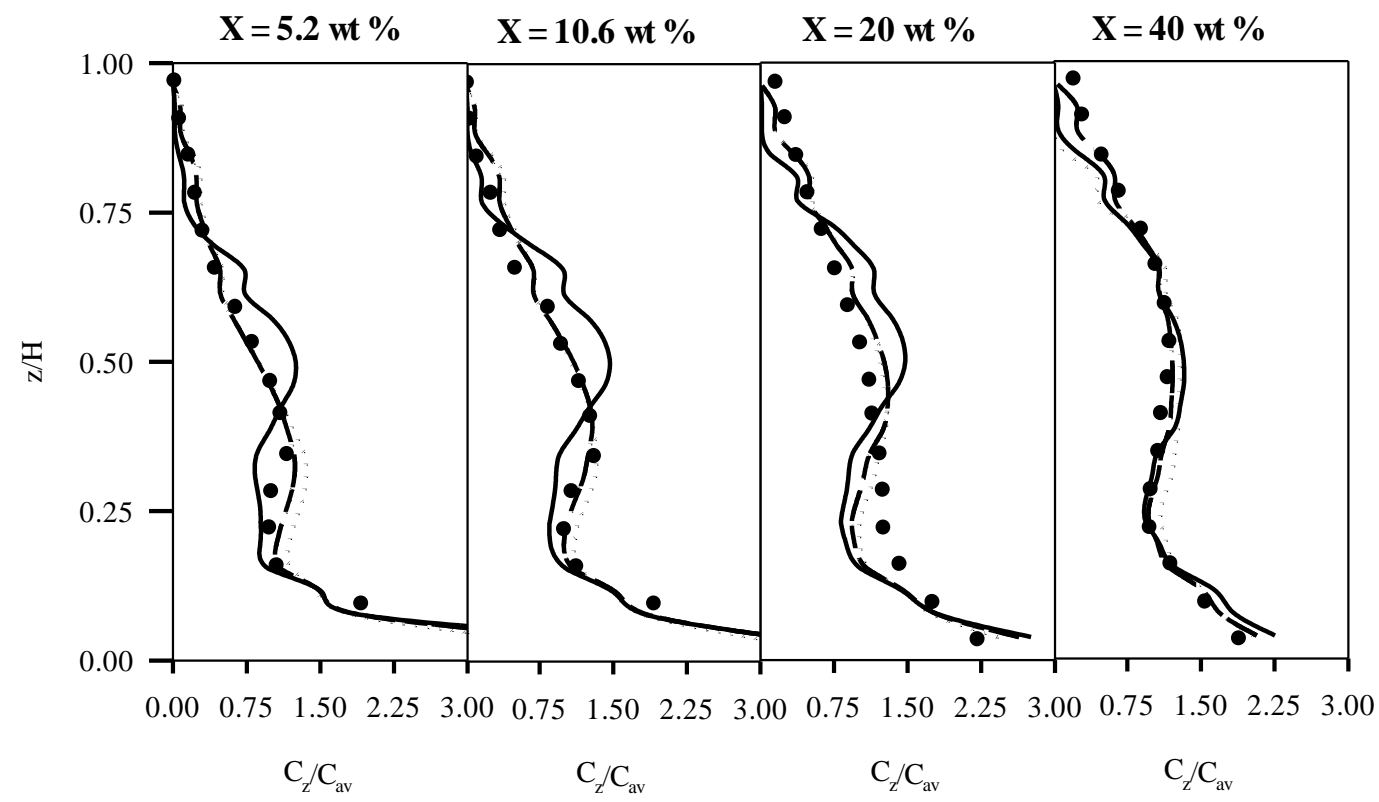

Figure 13. Azimuthally and radially averaged normalized solid concentrations plotted axially at $\mathrm{N}=\mathrm{Njs}$. 
- Experiment $\longrightarrow$ No Dispersion $\ldots . .$. DBL $\quad-$ Simonin - $\cdots-\cdots \quad$ Burns et al.

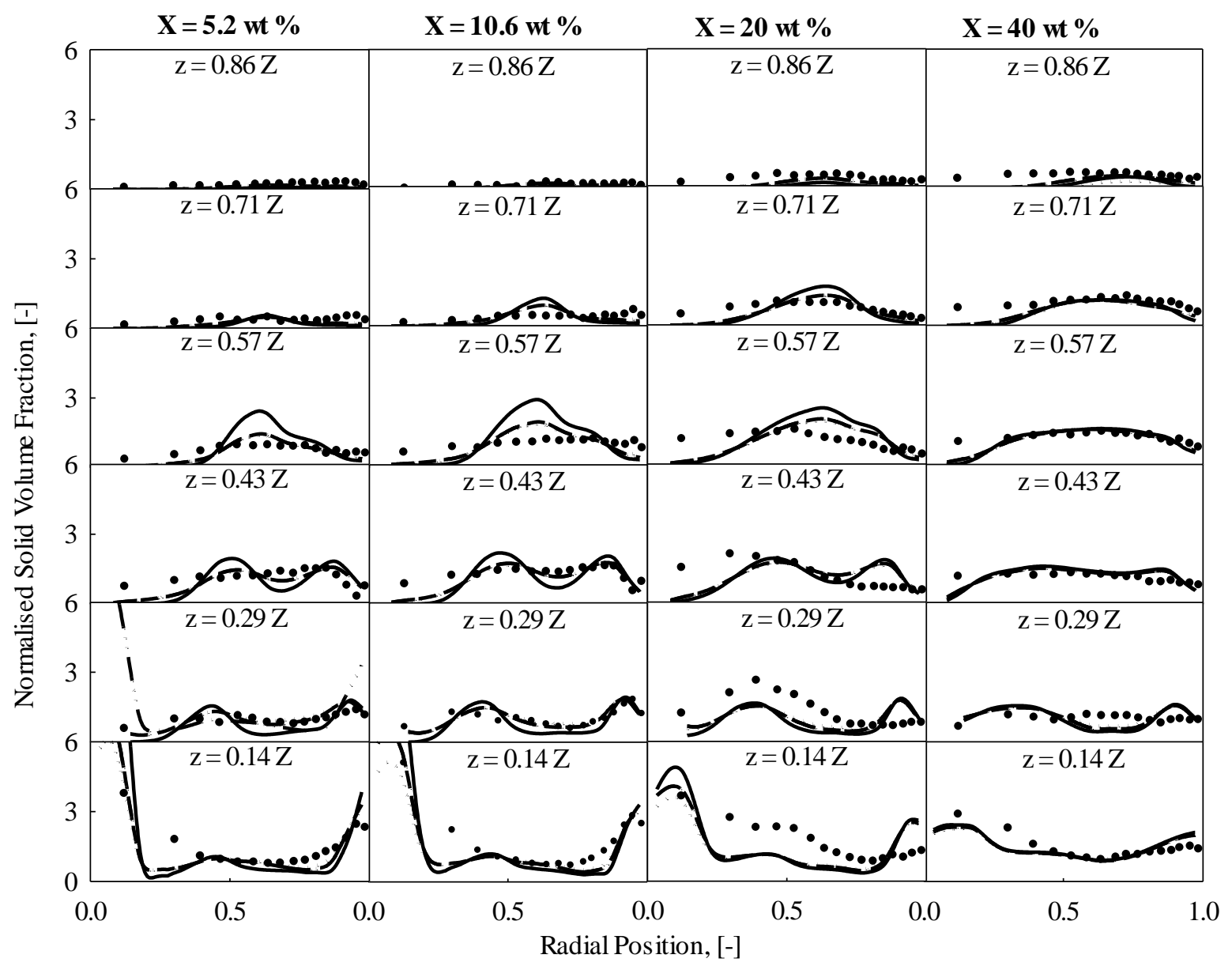

Figure 14. Circumferentially averaged normalized radial concentrations profiles at various heights at $\mathrm{N}=\mathrm{Njs}$.

Azimuthally and radially averaged values of dispersion force using these three models are plotted against the height of the stirred tanks (Figure 12). Values from Simonin and Burns models predict nearly similar values for the force at all solid concentrations. However, the DBL model gives double the values compared to other two models at low solid concentration and comparable values at intermediate concentration (20 wt \%). At very high solid concentration, DBL gives low values near the top of the tank and high values near the bottom. Nevertheless, the suitability of the dispersion force model can only be confirmed by applying it to the simulations and comparing the concentration predictions with experimental values. When the turbulent dispersion force was included in the calculations, the predictions improved for all the cases (see Figure 13 and Figure 14). Specifically, the error in the predictions observed while comparison of drag models near the top of tanks is diminished with the application of this force. Furthermore, the overshoot in values at the height of $0.57 \mathrm{z}$ also suppressed. DBL model used in the paper uses a constant value of 1.0 for the turbulent dispersion constant, which is not applicable with varying flow regimes in the stirred tanks. The impact is visible at solid loading of $40 \mathrm{wt} \%$ where it fails to predict dispersion effectively. Both Simonin et al. and Burns et al. model provided similar results due to identical values of dispersion scalar. Therefore, either of these models can be used to model dispersion in stirred tanks. In this study, Burns et al. model is used for the further investigation of turbulence model. 


\section{Turbulence Models}

k- $\varepsilon$ turbulence model is the most widely used turbulence model for RANS simulation of stirred tanks. For multiphase modelling, k- $\varepsilon$ mixture, dispersed and "each phase" models are available. In the current cases, results from the EE-KTGF simulations with these three turbulence models are compared. The fluctuating velocity (granular temperature) for secondary phases is calculated using KTGF. k- $\varepsilon$ mixture model and "each phase" models provide similar results. $\mathrm{k}-\varepsilon$ dispersed model is a modification of standard k- $\varepsilon$ model with modified Reynolds stress tensor. Its applicability is only limited to dilute flows. This is supported by the findings from simulations as it predicts reasonably well at low solid loading, but provides unrealistic results as the solid concentration increased. Similar observations were reported by G. Montante and Magelli (2005) who also analysed these turbulence models.

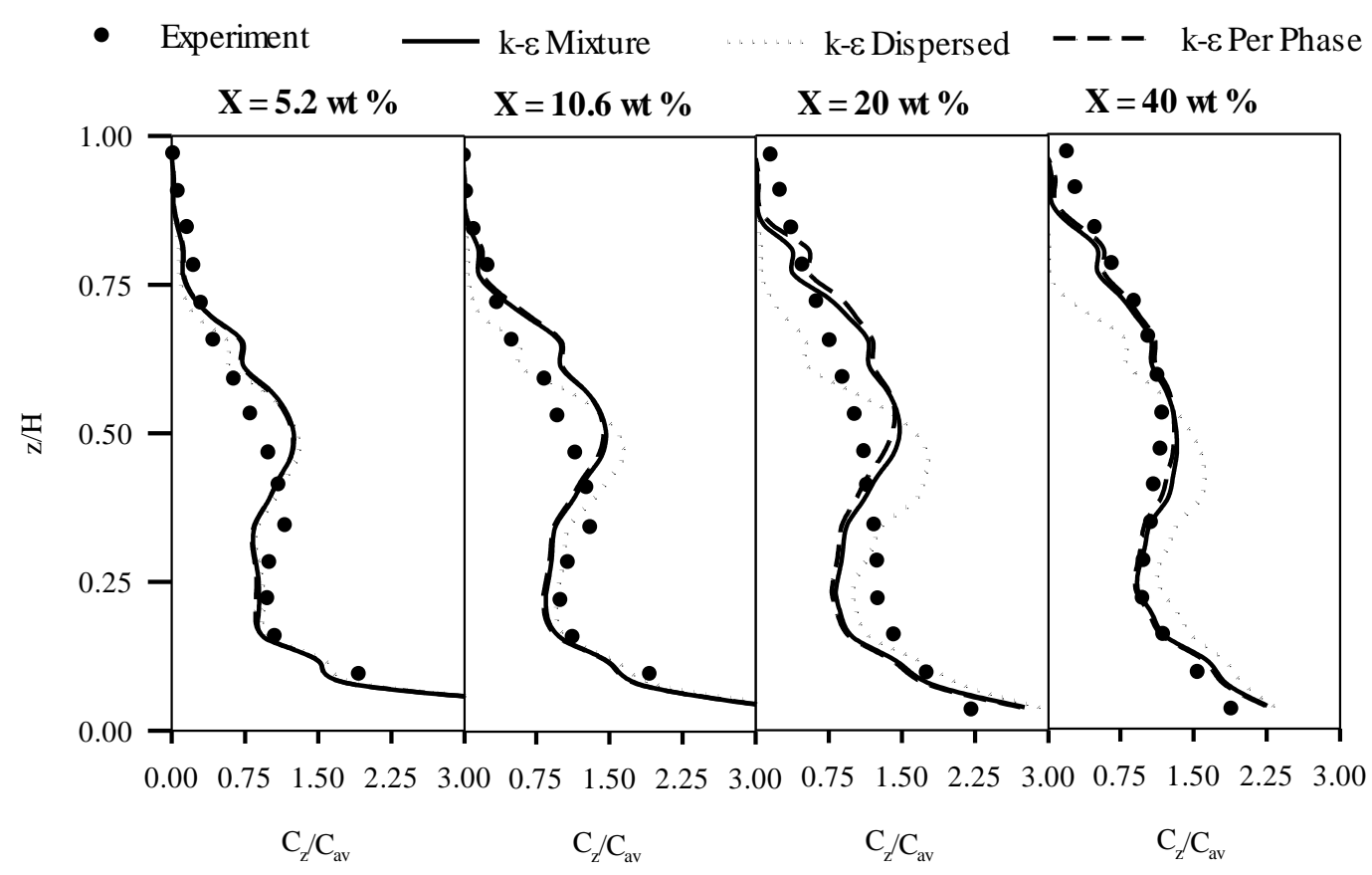

Figure 15 . Azimuthally and radially averaged normalized solid concentrations plotted axially at $\mathrm{N}=\mathrm{Njs}$.

Other than k- $\varepsilon$ mixture, k- $\varepsilon$ Re-normalised Group (RNG), k- $\varepsilon$ Realizable, k- $\omega$ Standard, k- $\omega$ Shear Stress Transport (SST) and Reynolds Stress Model (RSM) are also available that can be used for RANS simulations. The normalised axial and radial concentration profiles from the simulation results obtained using these models are plotted in Figure 16 and Figure 17. The variation in the prediction is insignificant at high solid loading of $40 \mathrm{wt} \%$. But as the solid loading decreases, the variation becomes evident. k- $\varepsilon$ standard model finds its limitation in under-predicting the turbulent kinetic energy (Aubin et al., 2004). The error in the predictions due to this model can be attributed to the flows near stirrer blades.

k- $\varepsilon$ RNG model simulates turbulence while eliminating small scale eddies which are essential in the region around impeller. This elimination improves the accuracy of low Reynolds number rapidly strained flows, but is undesirable for highly turbulent flows in stirred tanks. With k-E RNG models, the turbulence is grossly 
under-predicted by more than an order of magnitude in the highly turbulent lower half of tank and is overpredicted near the top in the low Reynolds number region. Furthermore, the turbulence dissipation rate using $\mathrm{k}-\varepsilon$ RNG models is also $30 \%$ of $\mathrm{k}-\varepsilon$ standard model in the impeller discharge region and an order of magnitude higher at the top centre of the tank.

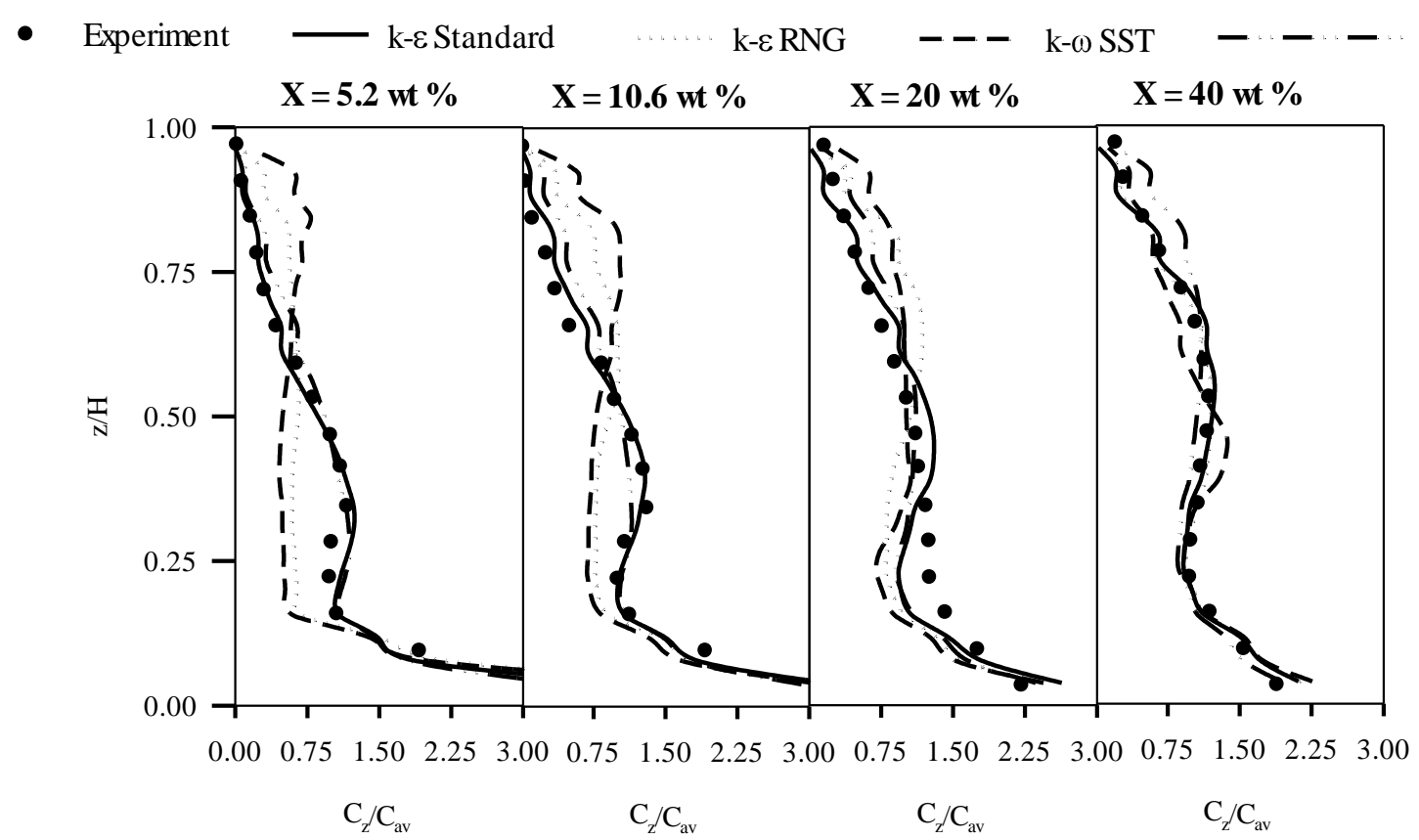

Figure 16. Azimuthally and radially averaged normalized solid concentrations plotted axially at $\mathrm{N}=\mathrm{Njs}$.

The turbulence kinetic energy in k- $\varepsilon$ standard and k- $\varepsilon$ Realizable models is formulated using the same way. The difference is in the formulation of dissipation rate that satisfies the mathematical constraints viz. positivity of normal stresses and Schwarz inequality for shear stresses. Rather than using a constant value of 0.09, its value is kept variable across the domain. For the bulk of the fluid in stirred tanks, its value varies between 0.05 and 0.12 . For both the cases, the turbulent kinetic energy and dissipation rates are qualitatively the same with very little quantitative variation. The RMS error between standard and Realizable models for $\mathrm{k}$ and $\varepsilon$ are 0.03 and 1.15 respectively compared to RMS error between standard and RNG models for $\mathrm{k}$ and $\varepsilon$ of 0.08 and 4.03 respectively. Using the k- $\varepsilon$ Realizable model does not provide any advantage in local solid volume fraction predictions. Since, the lines for both the models almost overlap, only the results of k- $\varepsilon$ standard model are shown in Figure 16 and Figure 17.

k- $\omega$ standard and k- $\omega$ SST were formulated to model the low Reynolds number flows in the vicinity of walls. Instead of dissipation rate, equations for specific dissipation rate are used in the models. Low Reynolds number correction is employed in the turbulence viscosity by dampening it with a factor based on turbulent Reynolds number. As a result, the value of turbulent viscosity is decreased by four folds in the bulk of fluid and holds an average value of $0.4 \mathrm{~kg} /(\mathrm{m} . \mathrm{s})$ compared to $1.8 \mathrm{~kg} /(\mathrm{m} . \mathrm{s})$ for the $\mathrm{k}-\varepsilon$ standard case. Moreover, its value diminishes significantly at the tank bottom centre. The values of $\mathrm{k}$ and $\varepsilon$ also follow the same course and have far lower values. The severe under-prediction in turbulence values results in the predictions that are worse 
than those of k- $\varepsilon$ RNG model for low solid volume fractions. At higher solid volume fractions, the k- $\omega$ models predict higher turbulence at top compared to the bulk region that assists in suspension of solids at the top of stirred tanks. However, it should be noted that the turbulence predictions differ by two orders of magnitudes at high solid concentration. The error in the predictions is not visible as it is suppressed with the normalization of concentration profiles by average high concentration. In all the cases studied in the paper, the concentration values predicted by k- $\omega$ standard and k- $\omega$ SST models are similar and therefore, only the results of k- $\omega$ SST model are shown in Figure 16 and Figure 17.

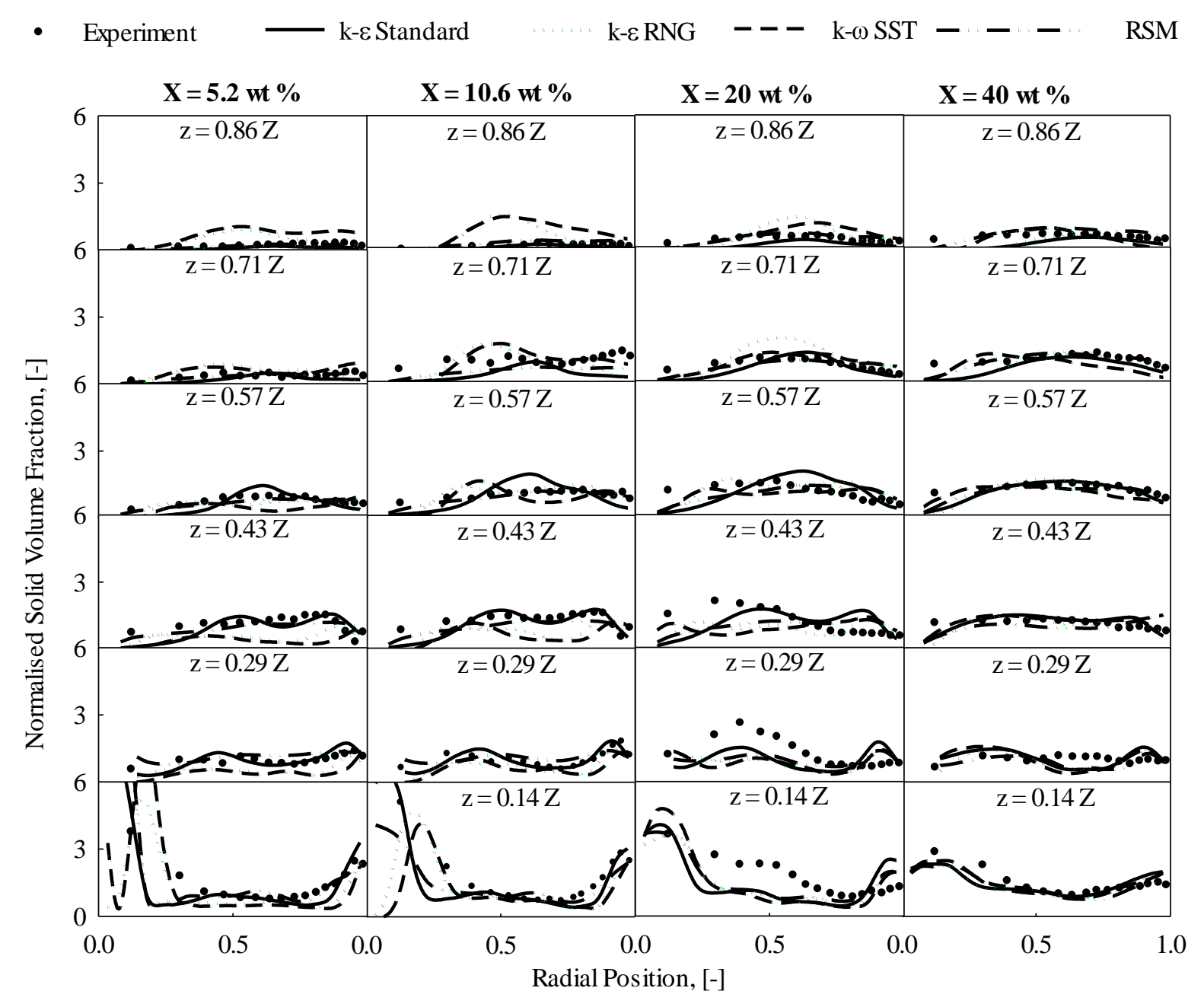

Figure 17. Circumferentially averaged normalized radial concentrations profiles at various heights at $\mathrm{N}=\mathrm{Njs}$.

RSM has proved advantageous in the case of stirred tanks where the flow imparted by the impeller can produce anisotropic turbulence. Therefore, modelling it is necessary for accurate local predictions in stirred tanks. Although this model is computationally expensive as it solves separate equations to resolve the Reynolds stresses, but the desired accuracy is visible in both radial concentration plots shown in Figure 16. The overpredictions in the axial concentration profiles observed in Figure 17 are a cumulative effect of marginal improvement seen in radial concentration profiles. Furthermore, the kinetic energy in the impeller discharge regions for the k- $\varepsilon$ standard and RSM model are compared and it is found that its value is 5 to 6 times higher. Another region that shows disagreement between these models is the top centre region of stirred tank, where 
the flow is decelerating and the turbulent kinetic energy is half of that observed in RSM. k- $\varepsilon$ standard severely under-predicts the solid concentration in this region, where the predictions of RSM model prove fairly well and have less than $5 \%$ error compared to experimental data. In both the cases, the maximum turbulence dissipation occurs at the impeller. But majority of turbulence dissipation is limited to the lower half of the stirred tank for the k- $\varepsilon$ model. While in the case of RSM model, turbulence dissipation is extended to the top of the stirred tank through the region between impeller rod and tank wall. These factors have led to improved predictions of solids suspension near the top of tanks using RSM model.

\section{Conclusion}

The accuracy of the simulation results for solids suspension in low to high solids concentration in stirred tanks systems is dependent on various constitutive models. Limited experimental data is available for high solids concentration. Similarly, there is lack of consensus on appropriate constitutive models for high solids concentration. In the present work, a detailed investigation of the modelling approaches, particle-particle interactions, drag models, turbulence dispersion models and turbulence models is presented. The conclusions deduced are as follows:

1. The flow field generated using EE approach and EE-KTGF approach do not differ at low solid concentrations as particle-particle interactions are minimal. At high solid loading, the EE-KTGF approach predicts the diversion of jet before hitting the bottom of the stirred tank accurately, which is not visible in the results using EE approach. While EE-KTGF approach is more appropriate, it grossly over-predicts at the bottom centre and under-predicts near the top of the tank in the absence of appropriate constitutive models.

2. Increasing the coefficient of restitution from 0.9 to 0.99 , the percent suspension increased from 98.2 $\%$ to $99.4 \%$. The use of high value of restitution coefficient $(=0.99)$ adversely affects the results in the impeller plane of the stirred tank, where solid concentration is over-predicted. The intermediate value of 0.95 for restitution coefficient appears to represent the system more closely compared to 0.9 and 0.99 .

3. At the lower solid concentrations, the predictions using modified Brucato drag model are eccentric. The error reduces substantially with concentration increase to $20 \mathrm{wt} \%$ and $40 \mathrm{wt} \%$ as in these cases the continuity assumption for the solid phase is satisfied. At $40 \mathrm{wt} \%$, predictions of Huilin-Gidaspow, Tenneti and Syamlal \& Brien drag model are in reasonable agreement with the experimental data. Syamlal drag model maintains its efficiency upto a height of $0.43 \mathrm{z}$, above which it under-predicts the solid concentration values at higher solid loading and results in an overshoot at $0.6 \mathrm{R}$ for low solid concentration. However, compared to the other models, the error in predictions using Syamlal drag model is minimal for the simulation of solid loading stirred tanks over the range of solid volume fraction studied in the paper. 
4. Values from Simonin and Burns models predict nearly similar values for the turbulent dispersion force at all solid concentrations. However, the DBL model gives double the values compared to other two models at low solid concentration and comparable values at intermediate concentration (20 wt \%). The error in the predictions near the top of tanks observed is diminished with the application of this force. Furthermore, the overshoot in solid concentration values at the height above $0.43 \mathrm{z}$ is also suppressed. At solid loading of $40 \mathrm{wt} \%$, DBL model fails to predict dispersion effectively and both Simonin et al. and Burns et al. model provided accurate results. Either of these models can be used to model turbulent dispersion in stirred tanks.

5. k- $\varepsilon$ mixture model and "each phase" models provided similar results with error in predictions lower than $\mathrm{k}-\varepsilon$ dispersed model. $\mathrm{k}-\varepsilon$ dispersed model predicted the solid concentration profiles reasonably well at low solid loading, but produced unrealistic results as the solid concentration increased. With k$\varepsilon$ RNG models, the turbulence is grossly under-predicted by more than an order of magnitude in the highly turbulent lower half of tank and is over-predicted near the top in the low Reynolds number region. Similarly, the k- $\varepsilon$ Realizable model also does not provide any advantage in local solid volume fraction predictions. The predictions further deteriorated using k- $\omega$ standard and k- $\omega$ SST models as the value of turbulent viscosity decreased by four folds in the bulk of fluid and holds an average value of $0.4 \mathrm{~kg} /(\mathrm{m} . \mathrm{s})$ compared to $1.8 \mathrm{~kg} /(\mathrm{m} . \mathrm{s})$ for the $\mathrm{k}-\varepsilon$ standard case. The values of $\mathrm{k}$ and $\varepsilon$ also follow the same course and have far lower values. RSM has proved advantageous in the case of stirred tanks where the flow imparted by the impeller can produce anisotropic turbulence. Although this model is computationally expensive as it solves separate equations to resolve the Reynolds stresses, but the desired accuracy is achieved in predicting the local hydrodynamics in the stirred tanks.

\section{References}

Altway, A., Setyawan, H., Margono, \& Winardi, S. (2001). Effect of Particle Size on Simulation of ThreeDimensional Solid Dispersion in Stirred Tank. Chemical Engineering Research and Design, 79(8), 10111016.

Aubin, J., Fletcher, D. F., \& Xuereb, C. (2004). Modeling turbulent flow in stirred tanks with CFD: the influence of the modeling approach, turbulence model and numerical scheme. Experimental Thermal and Fluid Science, 28(5), 431-445.

Barigou, M. (2004). Particle Tracking in Opaque Mixing Systems: An Overview of the Capabilities of PET and PEPT. Chemical Engineering Research and Design, 82(9), 1258-1267.

Beetstra, R., van der Hoef, M. A., \& Kuipers, J. A. M. (2007). Drag force of intermediate Reynolds number flow past mono- and bidisperse arrays of spheres. AIChE Journal, 53(2), 489-501. 
Bittorf, K. J., \& Kresta, S. M. (2003). Prediction of Cloud Height for Solid Suspensions in Stirred Tanks. Chemical Engineering Research and Design, 81(5), 568-577.

Burns, A. D., Frank, T., Hamill, I., \& Shi, J.-M. (2004). The Favre averaged drag model for turbulent dispersion in Eulerian multi-phase flows. Paper presented at the 5th International Conference on Multiphase Flow, ICMF.

Cello, F., Di Renzo, A., \& Di Maio, F. P. (2010). A semi-empirical model for the drag force and fluid-particle interaction in polydisperse suspensions. Chemical Engineering Science, 65(10), 3128-3139.

Crowe, C. T. (2000). On models for turbulence modulation in fluid-particle flows. International Journal of Multiphase Flow, 26(5), 719-727.

de Bertodano, M. L., Lahey Jr, R. T., \& Jones, O. C. (1994). Turbulent bubbly two-phase flow data in a triangular duct. Nuclear Engineering and Design, 146(1-3), 43-52.

Derksen, J., Akker, v. d., \& Harry, E. A. (1999). Large eddy simulations on the flow driven by a Rushton turbine. AIChE Journal, 45(2), 209-221.

Derksen, J. J. (2003). Numerical simulation of solids suspension in a stirred tank. American Institute of Chemical Engineers. AIChE Journal, 49(11), 2700-2700.

Ergun, S. (1952). Fluid flow through packed columns. Chemical Engineering Progress, 48(2), 89-94.

Fan, L., Mao, Z., \& Wang, Y. (2005). Numerical simulation of turbulent solid-liquid two-phase flow and orientation of slender particles in a stirred tank. Chemical Engineering Science, 60(24), 7045-7056.

Felice, R. d. (1996). A relationship for the wall effect on the settling velocity of a sphere at any flow regime. International Journal of Multiphase Flow, 22(3), 527-533.

Feng, X., Li, X., Cheng, J., Yang, C., \& Mao, Z.-S. (2012). Numerical simulation of solid-liquid turbulent flow in a stirred tank with a two-phase explicit algebraic stress model. Chemical Engineering Science, 82, 272-284.

Fletcher, D. F., \& Brown, G. J. (2009). Numerical simulation of solid suspension via mechanical agitation: effect of the modelling approach, turbulence model and hindered settling drag law. International Journal of Computational Fluid Dynamics, 23(2), 173-187.

Fradette, L., Tanguy, P. A., Bertrand, F., Thibault, F., Ritz, J.-B., \& Giraud, E. (2007). CFD phenomenological model of solid-liquid mixing in stirred vessels. Computers \& Chemical Engineering, 31(4), 334-345.

Gibilaro, L., Di Felice, R., Waldram, S., \& Foscolo, P. (1985). Generalized friction factor and drag coefficient correlations for fluid-particle interactions. Chemical Engineering Science, 40(10), 1817-1823. 
Gidaspow, D. (1994). Multiphase flow and fluidization: continuum and kinetic theory descriptions. San Diego: Academic Press.

Gohel, S., Joshi, S., Azhar, M., Horner, M., \& Padron, G. (2012). CFD Modeling of Solid Suspension in a Stirred Tank: Effect of Drag Models and Turbulent Dispersion on Cloud Height. International Journal of Chemical Engineering, 2012, 9.

Goldschmidt, M. J. V., Kuipers, J. A. M., \& van Swaaij, W. P. M. (2001). Hydrodynamic modelling of dense gas-fluidised beds using the kinetic theory of granular flow: effect of coefficient of restitution on bed dynamics. Chemical Engineering Science, 56(2), 571-578.

Guida, A., Nienow, A. W., \& Barigou, M. (2010). PEPT measurements of solid-liquid flow field and spatial phase distribution in concentrated monodisperse stirred suspensions. Chemical Engineering Science, 65(6), 1905-1914.

Huilin, L., Gidaspow, D., Bouillard, J., \& Wentie, L. (2003). Hydrodynamic simulation of gas-solid flow in a riser using kinetic theory of granular flow. Chemical Engineering Journal, 95(1-3), 1-13.

Kasat, G. R., Khopkar, A. R., Ranade, V. V., \& Pandit, A. B. (2008). CFD simulation of liquid-phase mixing in solid-liquid stirred reactor. Chemical Engineering Science, 63(15), 3877-3885.

Khopkar, A. R., Kasat, G. R., Pandit, A. B., \& Ranade, V. V. (2006). Computational Fluid Dynamics Simulation of the Solid Suspension in a Stirred Slurry Reactor. Industrial and Engineering Chemistry Research, 45(12), 4416-4428.

Liu, L., \& Barigou, M. (2013). Numerical modelling of velocity field and phase distribution in dense monodisperse solid-liquid suspensions under different regimes of agitation: CFD and PEPT experiments. Chemical Engineering Science, 101(0), 837-850.

Ljungqvist, M., \& Rasmuson, A. (2001). Numerical Simulation of the Two-Phase Flow in an Axially Stirred Vessel. Chemical Engineering Research and Design, 79(5), 533-546.

Micale, G., Grisafi, F., Rizzuti, L., \& Brucato, A. (2004). CFD Simulation of Particle Suspension Height in Stirred Vessels. Chemical Engineering Research and Design, 82(9), 1204-1213.

Micale, G., Montante, G., Grisafi, F., Brucato, A., \& Godfrey, J. (2000). CFD Simulation of Particle Distribution in Stirred Vessels. Chemical Engineering Research and Design, 78(3), 435-444.

Montante, G., \& Magelli, F. (2005). Modelling of solids distribution in stirred tanks: analysis of simulation strategies and comparison with experimental data. International Journal of Computational Fluid Dynamics, 19(3), 253-253. 
Montante, G., Rondini, D., Bakker, A., \& Magelli, F. (2002). CFD predictions of solid concentration distributions in a baffled stirred vessel agitated with multiple PBT impellers. Paper presented at the Proceedings of the CHISA Conference.

Murthy, B. N., \& Joshi, J. B. (2008). Assessment of standard, RSM and LES turbulence models in a baffled stirred vessel agitated by various impeller designs. Chemical Engineering Science, 63(22), 5468-5495.

Ochieng, A., \& Lewis, A. E. (2006a). CFD simulation of solids off-bottom suspension and cloud height. Hydrometallurgy, 82(1-2), 1-12.

Ochieng, A., \& Lewis, A. E. (2006b). Nickel solids concentration distribution in a stirred tank. Minerals Engineering, 19(2), 180-189.

Ochieng, A., \& Onyango, M. S. (2008). Drag models, solids concentration and velocity distribution in a stirred tank. Powder Technology, 181(1), 1-8.

Oshinowo, L. M., \& Bakker, A. (2002). CFD modeling of solids suspensions in stirred tanks. Paper presented at the Proceedings of the Conference on Computational Modeling of Materials, Minerals and Metals Processing.

Pianko-Oprych, P., Nienow, A. W., \& Barigou, M. (2009). Positron emission particle tracking (PEPT) compared to particle image velocimetry (PIV) for studying the flow generated by a pitched-blade turbine in single phase and multi-phase systems. Chemical Engineering Science, 64(23), 4955-4968.

Ranade, V. V. (2001). Computational flow modeling for chemical reactor engineering (Vol. 5). San Diego: Academic press.

Rong, L. W., Dong, K. J., \& Yu, A. B. (2013). Lattice-Boltzmann simulation of fluid flow through packed beds of uniform spheres: Effect of porosity. Chemical Engineering Science, 99(0), 44-58.

Sardeshpande, M. V., Juvekar, V. A., \& Ranade, V. V. (2010). Hysteresis in cloud heights during solid suspension in stirred tank reactor: Experiments and CFD simulations. AIChE Journal, 56(11), 2795-2804.

Sardeshpande, M. V., Sagi, A. R., Juvekar, V. A., \& Ranade, V. V. (2009). Solid Suspension and Liquid Phase Mixing in Solid-Liquid Stirred Tanks. Industrial and Engineering Chemistry Research, 48(21), 9713-9713.

Simonin, O., \& Viollet, P. (1990). Modelling of turbulent two-phase jets loaded with discrete particles. Phenomena in Multiphase Flow, 259-269.

Stevenson, R., Harrison, S. T. L., Mantle, M. D., Sederman, A. J., Moraczewski, T. L., \& Johns, M. L. (2010). Analysis of partial suspension in stirred mixing cells using both MRI and ERT. Chemical Engineering Science, 65(4), 1385-1393.

Syamlal, M., Rogers, W., \& O’Brien, T. J. (1993). MFIX documentation: Theory guide. Technical Note, DOE/METC-94/1004, NTIS/DE94000087, National Technical Information Service, Springfield, VA. 
Tamburini, A., Cipollina, A., Micale, G., Brucato, A., \& Ciofalo, M. (2011). CFD simulations of dense solidliquid suspensions in baffled stirred tanks: Prediction of suspension curves. Chemical Engineering Journal, 178(0), 324-341.

Tamburini, A., Cipollina, A., Micale, G., Brucato, A., \& Ciofalo, M. (2012). CFD simulations of dense solidliquid suspensions in baffled stirred tanks: Prediction of the minimum impeller speed for complete suspension. Chemical Engineering Journal, 193-194(0), 234-255.

Tamburini, A., Cipollina, A., Micale, G., Brucato, A., \& Ciofalo, M. (2014). Influence of drag and turbulence modelling on CFD predictions of solid liquid suspensions in stirred vessels. Chemical Engineering Research and Design, 92(6), 1045-1063.

Tamburini, A., Cipollina, A., Micale, G., Ciofalo, M., \& Brucato, A. (2009). Dense solid-liquid off-bottom suspension dynamics: Simulation and experiment. Chemical Engineering Research and Design, 87(4), 587597.

Tenneti, S., Garg, R., \& Subramaniam, S. (2011). Drag law for monodisperse gas-solid systems using particleresolved direct numerical simulation of flow past fixed assemblies of spheres. International Journal of Multiphase Flow, 37(9), 1072-1092.

Unadkat, H., Rielly, C. D., Hargrave, G. K., \& Nagy, Z. K. (2009). Application of fluorescent PIV and digital image analysis to measure turbulence properties of solid-liquid stirred suspensions. Chemical Engineering Research and Design, 87(4), 573-586.

Wadnerkar, D., Utikar, R. P., Tade, M. O., \& Pareek, V. K. (2012). CFD simulation of solid-liquid stirred tanks. Advanced Powder Technology, 23(4), 445-453.

Wen, C. Y., \& Yu, Y. H. (1966). Mechanics of Fluidization.

Yamazaki, H., Tojo, K., \& Miyanami, K. (1986). Concentration profiles of solids suspended in a stirred tank. Powder Technology, 48(3), 205-216.

Zwietering, T. N. (1958). Suspending of solid particles in liquid by agitators. Chemical Engineering Science, 8(3-4), 244-253. 